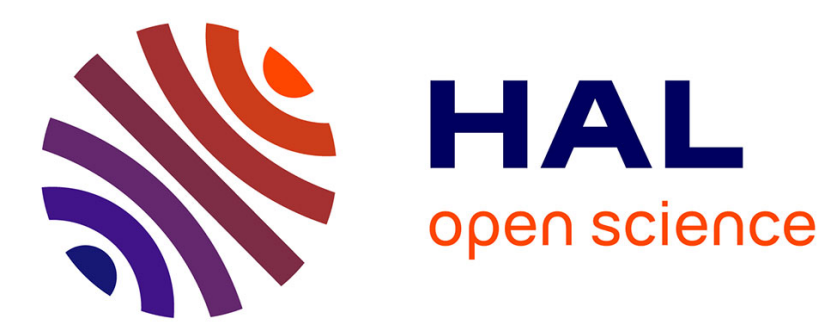

\title{
La rationalisation des méthodes d'évaluation des risques de récidive
}

Émilie Dubourg, Virginie Gautron

\section{To cite this version:}

Émilie Dubourg, Virginie Gautron. La rationalisation des méthodes d'évaluation des risques de récidive. Champ Pénal, 2014, Vol. XI, 10.4000/champpenal.8947. hal-02320993

\section{HAL Id: hal-02320993 \\ https://hal.science/hal-02320993}

Submitted on 20 Oct 2019

HAL is a multi-disciplinary open access archive for the deposit and dissemination of scientific research documents, whether they are published or not. The documents may come from teaching and research institutions in France or abroad, or from public or private research centers.
L'archive ouverte pluridisciplinaire HAL, est destinée au dépôt et à la diffusion de documents scientifiques de niveau recherche, publiés ou non, émanant des établissements d'enseignement et de recherche français ou étrangers, des laboratoires publics ou privés. 
Parentalités enfermées/Objets et enfermement/Probation française

Émilie Dubourg et Virginie Gautron

\title{
La rationalisation des méthodes d'évaluation des risques de récidive Entre promotion institutionnelle, réticences professionnelles et prudence interprétative
}

\begin{abstract}
Avertissement
Le contenu de ce site relève de la législation française sur la propriété intellectuelle et est la propriété exclusive de l'éditeur.

Les œuvres figurant sur ce site peuvent être consultées et reproduites sur un support papier ou numérique sous réserve qu'elles soient strictement réservées à un usage soit personnel, soit scientifique ou pédagogique excluant toute exploitation commerciale. La reproduction devra obligatoirement mentionner l'éditeur, le nom de la revue, l'auteur et la référence du document.

Toute autre reproduction est interdite sauf accord préalable de l'éditeur, en dehors des cas prévus par la législation en vigueur en France.
\end{abstract}

\section{revues.org}

Revues.org est un portail de revues en sciences humaines et sociales développé par le Cléo, Centre pour l'édition électronique ouverte (CNRS, EHESS, UP, UAPV).

\footnotetext{
Référence électronique

Éditeur : Association Champ pénal / Penal field http://champpenal.revues.org

http://www.revues.org

Document accessible en ligne sur :

http://champpenal.revues.org/8947

Document généré automatiquement le 18 décembre 2015.

(C) Champ pénal
}

Émilie Dubourg et Virginie Gautron, «La rationalisation des méthodes d'évaluation des risques de récidive », Champ pénal/Penal field [En ligne], Vol. XI | 2014, mis en ligne le 18 novembre 2014, consulté le 18 décembre 2015. URL : http://champpenal.revues.org/8947 ; DOI : 10.4000/champpenal.8947 
Émilie Dubourg et Virginie Gautron

\title{
La rationalisation des méthodes d'évaluation des risques de récidive
}

\author{
Entre promotion institutionnelle, réticences professionnelles et prudence \\ interprétative
}

\section{Introduction}

"Dans la première moitié du XIX siècle, une nouvelle attitude se fit jour. Le terme

de "science" fut de plus en plus restreint aux disciplines physiques et biologiques qui commencèrent au même moment à prétendre à une rigueur et à une certitude particulière qui les distingueraient de toutes les autres. Leur succès fut tel qu'elles en vinrent bientôt à exercer une extraordinaire fascination sur ceux qui travaillaient dans d'autres domaines ;

ils se mirent rapidement à imiter leur enseignement et leur vocabulaire. Ainsi débuta la

tyrannie que les méthodes et les techniques de la Science au sens étroit du terme n'ont jamais cessé d'exercer sur les autres disciplines. Celles-ci se soucièrent de plus en plus de revendiquer leur égalité de statut en montrant qu'elles adoptaient les mêmes méthodes que leurs sœurs dont la réussite était si brillante, au lieu d'adapter davantage leurs méthodes à leurs problèmes. Cette ambition d'imiter la Science dans ses méthodes plus que dans son esprit allait, pendant quelque cent vingt ans, dominer l'étude de l'homme, mais elle a dans le même temps à peine contribué à la connaissance des phénomènes sociaux; elle continue

cependant non seulement à dérouter et à discréditer les travaux des disciplines sociales, mais on présente encore toute demande de progrès nouveau dans cette direction comme la dernière innovation révolutionnaire capable de leur assurer - si on l'adoptait-une avance rapide et insoupçonnée. »(Hayek, 1953, 8).

L'individualisation de la peine constitue l'un des principes fondamentaux du droit pénal français (Saleilles, 1898). Légalement consacré, il enjoint les magistrats à tenir compte de la personnalité et de la situation personnelle du justiciable tant au stade de la détermination de la peine (art. 132-24 CP) que de ses modalités d'exécution (art. $707 \mathrm{CPP}$ ). À cette fin, différents professionnels, experts psychiatres et psychologues, conseillers pénitentiaires d'insertion et de probation, professionnels d'associations socio-judiciaires, interviennent tout au long du processus pénal afin de transmettre aux magistrats des éléments de connaissance sur la personnalité, la situation personnelle, socio-économique, professionnelle et sanitaire du justiciable. Toutefois, la rareté des enquêtes sociales rapides au stade pré-sentenciel (Danet, 2013) et les contraintes matérielles qui pèsent sur leur réalisation (Bernat de Celis, 1980 ; Tabary, 2005) compromettent l'idéal d'individualisation du prononcé de la peine. Ce principe se trouve reporté sur les modalités d'exécution de la sanction, en particulier par le biais de l'évaluation post-sentencielle des justiciables par les conseillers pénitentiaires d'insertion et de probation (CPIP), ce qui constituera la trame principale de cet article. L'exigence de célérité du procès pénal a en effet induit un déplacement de l'individualisation de la peine au stade de sa mise en œuvre concrète, la peine étant souvent exécutée sous des modalités très différentes de celles initialement prévues. Dans ce cadre, les finalités assignées à l'individualisation de la peine ont été sensiblement modifiées. Au vu des dernières évolutions législatives ${ }^{1}$ et réglementaires ${ }^{2}$, il s'agit moins d'individualiser la peine, afin de tendre à la réinsertion du justiciable, que de s'assurer que les mesures prononcées permettent de protéger efficacement la société du risque de récidive qu'il présente.

Cette évolution se dévoile notamment à travers le recours croissant aux expertises psychiatriques au stade post-sentenciel ${ }^{3}$. Les experts psychiatres sont ici mandatés afin non plus de se prononcer sur la responsabilité du justiciable ou sa réceptivité aux soins, mais davantage afin d'évaluer les risques de récidive, principalement des auteurs d'infractions à caractère sexuel ou violentes. L'expertise psychiatrique post-sentencielle répond ainsi à un objectif différent de l'expertise pré-sentencielle, conduisant les professionnels à établir, bien au-delà de leur mission expertale classique, la dangerosité criminologique d'individus qui ne 
présentent pas nécessairement une maladie psychiatrique stricto sensu (Manzanera, Senon, 2008). Mais ce déplacement est surtout notable au regard de la redéfinition des missions et méthodes d'intervention des Services pénitentiaires d'insertion et de probation (SPIP), dont la prévention de la récidive constitue désormais la finalité première ${ }^{4}$. Dans cette perspective, les CPIP doivent, au-delà d'une simple analyse des problématiques et situations individuelles, se prononcer sur le risque de récidive du justiciable en s'appuyant, comme le préconise le décret du 6 mai 2005, sur des connaissances criminologique ${ }^{5}$. Pour ce faire, l'administration pénitentiaire tente de redéfinir les méthodes d'évaluation des justiciables par les CPIP. Elle a ainsi doté ses agents d'un nouvel outil, le diagnostic à visée criminologique (DAVC), qui s'inscrivait dans cette nouvelle logique de gestion des risques. Le DAVC a été généralisé au sein des SPIP à compter du $1^{\text {er }}$ mars 2012, sur la base de la circulaire du 8 novembre 2011. Mais il a fait l'objet d'une mise en œuvre limitée, conduisant à son abandon progressif, entériné par l'annulation de la circulaire d'application par la décision du Conseil d'État du 11 avril 2014. Si la question de l'évaluation des justiciables est nouvellement saisie par les institutions, chercheurs et professionnels français, elle a fait l'objet de nombreuses recherches et suscite de vifs débats à l'étranger, principalement outre-Atlantique. Ces recherches ont conduit à l'élaboration d'outils d'évaluation structurés répondant à l'ambition d'une détermination précise des risques de récidive des justiciables. Il convient ainsi de replacer l'évolution des méthodes d'évaluation utilisées par les CPIP dans ce contexte international, afin de s'intéresser à ses enjeux en termes de positionnement professionnel des agents, mais également à ses effets sur la prise en charge des justiciables. Cet article se propose d'interroger la tentative de rationalisation des méthodes d'évaluation des justiciables dans un contexte de remise en cause des pratiques traditionnelles, essentiellement fondées sur une évaluation dite clinique. Nos investigations montrent que les professionnels français, de manière volontaire ou contrainte, sont conduits à amender progressivement leurs pratiques pour tenter d'objectiver les risques de récidive. L'introduction du DAVC au sein des SPIP a constitué un marqueur de cette évolution, soutenue par la Direction de l'Administration Pénitentiaire. Cet instrument d'évaluation des justiciables entendait répondre aux faiblesses du jugement professionnel des CPIP, reposant traditionnellement sur une approche clinique non structurée. Pour cela, le DAVC s'inspirait partiellement des méthodes actuarielles développées outre-Atlantique, méthodes qui reposent sur l'utilisation d'outils scorés permettant d'établir un niveau de risque de récidive. L'échec de cet instrument démontre néanmoins que les professionnels français demeurent réservés, sinon réticents, à l'idée d'adopter des outils standardisés, a fortiori scorés, au regard de leurs limites en termes de prise en charge des justiciables.

D'un point de vue méthodologique, notre propos s'appuie sur une revue de la littérature existante (rapports institutionnels, recherches scientifiques, textes à valeur juridique tant nationaux qu'étrangers) qui, sans prétendre à l'exhaustivité, traduit notre souci de présenter les différents positionnements, parfois antagonistes, qui se manifestent sur ce sujet. Cette étude se double d'une approche empirique des méthodes d'évaluation mises en œuvre par les CPIP. L'analyse s'appuie principalement sur les recherches doctorales d'Émilie Dubourg, fondées sur une enquête menée au sein de trois SPIP (Dubourg, s.d.). Ces services présentent l'intérêt d'être intégrés dans un environnement judiciaire, pénitentiaire et socio-économique différents, ce qui permet d'appréhender la diversité des pratiques et des modes d'organisations.

Tableau 1 : Caractéristiques des trois services étudiés

\begin{tabular}{|l|l|l|l|}
\hline & SPIP A & SPIP B & SPIP C \\
\hline Département & 800000 hab. & 650000 hab. & 600000 hab. \\
\hline Principale agglomération & 400000 hab. & 50000 hab. & 55000 hab. \\
\hline $\begin{array}{l}\text { Établissements } \\
\text { pénitentiaires }\end{array}$ & 1 maison d'arrêt & 2 maisons d'arrêt & $\begin{array}{l}1 \text { maison d'arrêt } \\
1 \text { centre de détention }\end{array}$ \\
\hline Personnels d'encadrement & 2 & 2 & 4 \\
\hline Personnels de probation & 24 & 17 & 32 \\
\hline Personnels administratifs & 3 & 3 & 4 \\
\hline
\end{tabular}


Au sein de ces services, différentes techniques d'enquêtes ont été mises en œuvre. Outre l'étude des rapports d'activité, une vingtaine d'entretiens semi-directifs ont été menés entre 2009 et 2012 auprès de différentes catégories de personnels (CPIP et personnels d'encadrement). Des entretiens ponctuels ont également été réalisés auprès de responsables de la Direction de l'Administration Pénitentiaire, de représentants des principaux syndicats et de magistrats (procureurs et juges de l'application des peines). Cette série d'entretiens a été complétée par des périodes d'observation et d'immersion au sein de deux des trois services. Dans ce cadre, des échanges plus informels se sont tenus, tant auprès des CPIP que des autres personnels pénitentiaires et judiciaires. Ces périodes d'observation ont permis de mieux appréhender le mode de fonctionnement des services ainsi que les relations entre les personnels et l'encadrement. Elles ont surtout été l'occasion d'assister à différents entretiens afin de mieux cerner toutes les facettes du métier de CPIP mais également la diversité des pratiques. Au sein du SPIP A, cette seconde phase s'est déroulée entre mai et juin 2012, à raison de trois à cinq jours par semaine. Six personnels d'insertion et probation et un personnel de surveillance ont pu être suivis dans leurs pratiques quotidiennes, au siège du service, dans le cadre de permanences délocalisées ou au sein des établissements pénitentiaires. Au sein du SPIP B, cette période d'observation a été plus restreinte, se concentrant sur cinq jours répartis entre janvier et mars 2012.

\section{I - Les méthodes classiques d'évaluation des justiciables: une approche qualitative désavouée}

Les professionnels français, et notamment les CPIP, recourent traditionnellement, et encore majoritairement, à des méthodes qualitatives pour évaluer le profil et la situation des justiciables et répondre à la question prospective du risque de récidive que ces derniers présentent. Usant de méthodes cliniques, ils s'appuient sur leur jugement professionnel, formulé à l'issue d'entretiens individuels souvent faiblement structurés. Les enseignements tirés de recherches et de pratiques étrangères ont nourri une contestation croissante de ces méthodes d'évaluation, les approches cliniques, jugées partiales, peu fiables ayant été désavouées au profit d'outils structurés, actuariels ou semi-actuariels.

\section{1) La prépondérance du jugement professionnel non structuré : une fiabilité contestée}

Les SPIP interviennent en milieu ouvert et en milieu fermé pour mettre à exécution la peine prononcée par les autorités judiciaires. La prise en charge des condamnés repose sur une évaluation préalable de leur profil, de leur situation personnelle, qui se prolonge tout au long de la mise en œuvre de la mesure. Cette évaluation permet de s'assurer de la pertinence du suivi, d'en rendre compte au magistrat mandant et de proposer, le cas échéant, une individualisation des mesures (art. D574 et D575 CPP). Dans ce cadre, les agents de probation mobilisent principalement leur jugement clinique formulé sur la base d'entretiens individuels. Ils s'appuient généralement sur une grille d'entretien élaborée localement au sein de chaque SPIP. Une relative homogénéité se dégage cependant de l'ensemble des trames étudiées. Elles reposent sur des items communs relatifs aux antécédents judiciaires, à la situation familiale, financière, professionnelle, sanitaire du justiciable. Cette trame ne constitue qu'un modèle, non contraignant, que les CPIP ne sont pas tenus de suivre. Ceux-ci disposent d'une liberté d'appréciation conséquente dans la manière dont ils conduisent leurs entretiens, abordent (ou non) les différents items et retranscrivent leurs observations, laissant ainsi la place à une certaine subjectivité de leurs évaluations. Le cadre de l'entretien individuel leur confère un important pouvoir discrétionnaire, qui se manifeste à la fois dans le choix des informations retenues et dans la façon de les combiner (Quirion, D’Addese, 2011, 234). Une grande disparité des pratiques s'observe ainsi. Certains agents se contentent de remplir la grille a minima, en recensant les informations à la manière d'un questionnaire administratif. Et si nombre d'entre eux complètent la grille par des notes inscrites sur papier libre, celles-ci ne sont pas toujours versées au dossier. Quelques agents, minoritaires, mobilisent des outils complémentaires pour affiner leur jugement face à certaines problématiques, notamment addictives ${ }^{6}$. Mais faute 
de disposer du temps et des moyens nécessaires, les agents ne vérifient que rarement les informations obtenues sur un mode purement déclaratif. Ainsi, alors que la visite à domicile représente l'un des moyens les plus pertinents pour prendre en compte l'environnement de la personne, aux plans matériel et relationnel (Dindo, 2011, 104) et impliquer l'entourage dans le processus de sortie de la délinquance, des considérations financières et sécuritaires ont justifié leur raréfaction ${ }^{7}$, y compris dans le cadre des enquêtes préalables à la mise en œuvre d'un Placement sous Surveillance Électronique (PSE). De même, l'évaluation de la situation sanitaire du justiciable et de son évolution, dans le cadre notamment d'une obligation de soins, se heurte au secret professionnel qui lie respectivement tant les professionnels de santé que les CPIP, les plaçant dans une forme d'ignorance mutuelle (Du Mesnil du Buisson, 2001, 4 ; v. également Lameyre, 2001). En outre, si les agents peuvent en principe s'appuyer sur les différentes pièces versées au dossier (expertises, jugement, extrait de casier judiciaire), les circuits de communication entre les différents services, notamment judiciaires et pénitentiaires, souffrent de faiblesses fréquemment soulignées, et ce en dépit de récents efforts en vue de leur dématérialisation (Blanc, 2007). Les logiciels utilisés tant par les CPIP que par les autorités judiciaires restent souvent cloisonnés (IGF, IGSJ, 2011). Ce cloisonnement contribue à placer les CPIP intervenant en détention en position de dépendance vis-à-vis du greffe pénitentiaire, ce qui nourrit certaines tensions locales. Les pièces judiciaires, quand elles sont fournies, constituent souvent les seuls éléments d'information extérieurs et a priori objectifs dont disposent les CPIP, générant une vision sélective et partielle du sujet (Ottenhof, Favard, 2001, 25). Ces différentes contraintes pèsent d'un poids particulier sur la qualité et la pertinence de l'évaluation, reposant essentiellement sur le jugement professionnel des agents, considéré, dans ces conditions, comme non structuré (Benbouriche et al., 2012). Les pratiques des agents sont perçues comme artisanales, lacunaires et perméables à une certaine subjectivité des propos sinon à des préjugés, en fonction de facteurs idiosyncrasiques liés à l'histoire, la formation, les valeurs et représentations des agents.

La fiabilité de ce type d'évaluations a été remise en cause. Les critiques visaient initialement les pratiques des experts psychiatres, qui s'appuyaient sur des méthodes d'évaluation cliniques non structurées. À partir des années 1960, des études anglo-saxonnes ont contesté la capacité de ces professionnels à évaluer la dangerosité, ou le risque de récidive, à partir de leur seul jugement clinique. Ce mouvement contestataire a été porté par John Monahan qui estima que l'évaluation de la dangerosité par un clinicien était exacte seulement une fois sur trois (Monahan, 1981), même s'il nuancera par la suite ses propos (Monahan, 1997). Des dizaines sinon des centaines d'études considèrent que les évaluations cliniques non structurées présentent des estimations proches du hasard, reprochent aux cliniciens une surévaluation des risques de récidive, la mobilisation de concepts psychanalytiques imprécis, sans rapport avec les comportements délictuels à prédire et détachés des classifications des maladies mentales, ce qui empêcherait toute reproductibilité et conduirait à une fidélité inter-juges peu élevée. À l'heure actuelle, et en dépit de ces critiques, les experts psychiatres français continuent d'accorder une place prépondérante à l'entretien clinique (Crampagne, 2013). Ces critiques ont été réactivées dans le domaine spécifique de la prise en charge des condamnés au début des années 1970. L'article publié en 1974 par le sociologue américain Robert Martinson, désormais résumé en deux mots Nothing Works, est considéré comme l'article fondateur de ce mouvement critique, l'auteur estimant que les méthodes de prise en charge alors mises en œuvre aux États-Unis n'avaient qu'une faible efficacité en termes de diminution des risques de récidive (Martinson, 1974). En réaction, des chercheurs principalement nordaméricains, rattachés au mouvement dit du What Works, tentent depuis les années 1990 de proposer des méthodes de prises en charge efficaces. Ils articulent leur réflexion autour de trois principes fondamentaux : le principe du risque, le principe du besoin et le principe de la réceptivité. Le principe central du risque suppose d'évaluer le risque de récidive au regard de facteurs de risque criminogènes, limitativement conçus (Bonta, 1997; Andrews, Bonta, 2007; v. également Sarre, 2001 ; Lalande, 2006).

10 Si la plupart des facteurs recherchés par les professionnels français, experts et agents de probation, sont des facteurs reconnus dans la littérature internationale comme étant des facteurs 
de risque (Crampagne 2013 ; Senon et al. (dir.), 2007), il leur est reproché de méconnaître les enseignements de la recherche sur les prédicteurs de risque, ce qui les conduirait à fonder leurs jugements sur des corrélations illusoires (Giovannangeli et al., 2000, 8). Il en va ainsi concernant la reconnaissance des faits par le condamné, le déni étant souvent considéré comme un facteur de risque par les médecins, les magistrats et les personnels d'insertion et de probation français. Or, des études internationales concluent à l'absence de corrélation entre cette variable et la récidive (Herzog-Evans, 2012b) ou à une corrélation prudente dépendant de la nature de la délinquance, du risque de récidive et de la forme de déni (Nunes et al., 2007 ; Lund, 2000). Les praticiens accorderaient également trop d'importance aux facteurs cliniques et comportementaux et sous-évalueraient les éléments de nature sociodémographique, environnementaux ou relevant de l'histoire du sujet, à l'exception de ceux concernant les violences antérieurement commises (Elbogen et al., 2002 ; Niveau, 2011). L'évaluation basée sur un jugement clinique non structuré serait donc impressionniste, subjective, non validée scientifiquement, et fondée sur des corrélations intuitives (Baratta, 2011, 11). Différents faits divers, impliquant des récidivistes, tels que l'affaire de Pornic ${ }^{9}$, ont également contribué à mettre en exergue la faiblesse de l'évaluation des agents de probation et leur incapacité à apprécier les risques de récidive (IGF-IGSJ, 2011 ; ISP, 2011). En réaction, la volonté politique et institutionnelle affichée de protéger la société contre les risques de récidive a conduit à une profonde remise en cause des méthodes de prise en charge des justiciables. Les CPIP français ont été invités, par leur Administration centrale, à se tourner vers des outils d'évaluation standardisés s'inspirant partiellement de ceux élaborés outre-Atlantique.

\section{2) Les instruments purement actuariels : une alternative quantitative présentée comme objective}

Dès le $\mathrm{XIX}^{\mathrm{e}}$ siècle, les nouveaux savoirs sur le crime ont été convoqués pour armer scientifiquement les décisions pénales (Kaluszynski, 2013). Dans Sociologie criminelle, Enrico Ferri (1914) prédisait le triomphe de ses idées dans un avenir qui ne lui semblait pas si éloigné. Il en déduisait le succès inéluctable de la force de son plus puissant allié, le fait, qui devait permettre la reconnaissance d'un nouvel esprit scientifique du droit criminel. À présent, les théories positivistes reviennent en force grâce à l'illusion d'une science criminologique qui aurait profondément évolué, quittant presque le domaine des sciences humaines pour rejoindre celui des sciences exactes. Reprenant à leur compte l'ambition initiale de Quételet, qui entendait dépouiller l'homme de son individualité pour éliminer tout ce qui est accidentel (cité in Ewald, 1996, 111), de nombreux chercheurs nord-américains ont développé des méthodes d'évaluation reposant sur des éléments objectifs appréhendés de manière statistique. À la suite des travaux de Burgess, Reiss, Ohlin, Glaser, Meelh ou encore des époux Glueck dans la première moitié du XX $\mathrm{X}^{\mathrm{e}}$ siècle (Harcourt, 2011a), et sous l'influence croissante du mouvement de la nouvelle pénologie (Feeley, Simon, 1992), des chercheurs se sont appuyés sur le développement des études consacrées aux facteurs de récidive pour construire de multiples échelles de prédiction des risques. Ces instruments s'appuient sur des méthodes statistiques inspirées des pratiques assurantielles, afin de déterminer les niveaux de risque associés à un groupe de délinquants présentant des caractéristiques communes, et, sur la base de ces corrélations, de prédire le comportement criminel futur d'un individu spécifique (Harcourt, 2011a). Ce faisant, ces outils établissent des liens de corrélation statistiques entre des facteurs et le risque de récidive, sans chercher à en expliquer les causes plus profondes (Guay, 2013), ce qui aux yeux de certains chercheurs, constitue un intérêt notable (Quinsey et al., 1998, 182). Ils font ainsi l'économie d'une analyse approfondie de la situation du délinquant pour ne s'intéresser qu'à certains facteurs, limitativement conçus, qualifiés de criminogènes. Certains de ces facteurs sont statiques, c'est-à-dire préexistants et imperméables à toute forme de prise en charge, comme le sexe, l'âge et les antécédents judiciaires, et d'autres sont dits dynamiques, c'est-à-dire susceptibles d'évolution, tels que la situation socioprofessionnelle, familiale, sanitaire ou l'environnement relationnel (Bonta, 1997 ; Andrews, Bonta, 2007). L'approche statistique de ces facteurs, mécanique et algorithmique (Groove et Meelh cités in Giovannangeli et al., 2000, 8 ; v. également Grove, Meelh, 1996), apparaît ainsi 
comme un gage de précision, d'uniformisation et d'efficacité (Quirion, D'Addese, 2011, 229). La prédiction actuarielle objective, valide et fidèle permettrait de dompter les incertitudes liées au comportement criminel (Proulx, Lussier, 2001, 19 ; Deprins, 2006, 510).

On estime à plus d'une centaine le nombre d'instruments d'évaluation des risques actuellement utilisés (Singh, Fazel, 2010). Malgré l'absence de consensus sur ce point (Hanson, 2010), de nombreuses recherches sur l'évaluation des risques tendent à démontrer que les prédicteurs de risque diffèrent selon la nature de la délinquance (sexuelle, violente, etc.) et/ou les caractéristiques des délinquants (femmes, adolescents, etc.). Élaborées dans les années 1990, les premières échelles, dites de seconde génération, portaient sur un nombre restreint de variables, essentiellement historiques et statiques. Parmi celles-ci, l'échelle Static-99 élaborée en 1999, puis révisée en 2002, par les chercheurs canadiens K. Hanson et D. Thornton constitue la plus couramment utilisée au Canada et aux États-Unis pour établir le risque de récidive sexuelle (Hanson, Thornton, 1999 ; Archer et al., 2006 ; Jackson, Hess, 2007 ; McGrath et al., 2010). Tout comme le VRAG (Violence Risk Apparaisal Guide), conçu par les chercheurs canadiens G. Harris, M. Rice et V. Quinsey en 1993 au sujet de la récidive violente, cet outil repose sur un système de cotation attribuant une note pour chaque facteur, le score final permettant d'établir la nature élevée ou faible du risque de récidive (Niveau, 2011). L'évaluation est conduite essentiellement à partir de la lecture du dossier du justiciable, sans qu'un entretien individuel ne soit nécessairement mené. Divers spécialistes ont toutefois pointé les limites des approches purement statiques (Niveau, 2011). Outre la prise en compte partielle des facteurs de récidive, ces instruments ne permettraient pas aux praticiens de formuler des observations cliniques fines, ni de tenir compte des fluctuations du niveau de risque dans le temps. Elles contribueraient, au contraire, à figer l'individu dans son comportement délinquant, en dépit des efforts et changements engagés dans le cadre de la prise en charge (Lussier, Davies, 2011 ; Senon, 2012 ; Guay, 2013). Elles ne permettraient pas non plus d'éclairer les professionnels sur les modes de prise en charge les plus judicieux, ni de comprendre la dynamique de l'acte infractionnel (Millaud, Dubreucq, 2012, 433). Si ces échelles sont encore utilisées aux États-Unis, au Canada et en Grande-Bretagne (HannahMoffat, 2013a, b), des chercheurs ont développé une troisième génération d'instruments, incluant, en parallèle des facteurs statiques, des variables dynamiques.

\section{3) Les instruments hybrides : un jugement professionnel structuré}

L'influence des facteurs dynamiques sur la récidive a été confirmée par plusieurs métaanalyses (Gendreau, Little, Goggin, 1996). Certaines de ces variables sont stables, fluctuant sur de relativement longues périodes et d'autres sont aiguës, c'est-à-dire brèves et situées dans une proximité temporelle avec l'acte infractionnel (Guay, 2013). Outre la Psychopathy ChecklistRevised (PCL-R) utilisée pour établir le niveau de personnalité antisociale (Hare, 1991), on compte parmi ces instruments, le Level of Service Inventory-Revised (LSI-R), élaboré par les chercheurs canadiens D. Andrews et J. Bonta en 1995 (Andrews, Bonta, Wormith, 2005) pour évaluer le risque de récidive générale, ainsi que les échelles Stable-2007 et Aigu-2007, utilisées pour évaluer le risque de récidive chez les auteurs d'infractions à caractère sexuel (Hanson et al., 2007). Cette approche a généré une nouvelle conceptualisation de la notion de dangerosité dans les années 1990. Celle-ci cesse alors d'être perçue comme constitutionnelle, statique et binaire (absente ou présente) mais apparaît davantage comme un phénomène contextuel, (dépendant de la situation et des circonstances), dynamique (sujet à changement) et continu (située dans un continuum de probabilité) (Niveau, 2011, 19). Ce changement de perspective se traduit par un glissement sémantique notable, le risque de récidive venant progressivement supplanter le terme de dangerosité au sein des évaluations. Alors que les échelles actuarielles de deuxième génération réduisent considérablement la marge d'appréciation des évaluateurs, l'approche dynamique réintroduit une part importante de jugement clinique (Côté, 2001, 41). Outre l'inclusion de critères cliniques et théoriques, non exclusivement sélectionnés sur une base probabiliste, ces outils ne donnent pas systématiquement lieu à un calcul actuariel, mais permettent un ajustement du résultat scoré sur la base d'observations cliniques. L'évaluation est ici conduite sous la forme d'entretiens structurés ou semi-structurés, complétés par la 
lecture du dossier du justiciable. Souvent assimilées à des outils actuariels dès lors qu'elles prennent la forme d'une grille d'évaluation automatisée et rigide, ces échelles sont davantage des instruments hybrides, qui combinent des caractéristiques de l'évaluation clinique et de l'évaluation actuarielle (Quirion, D'Addese, 2011).

Les instruments les plus récents, dits de quatrième génération, permettent d'approfondir l'analyse en proposant une évaluation intégrée. Outre l'appréciation du risque de récidive, ils permettent d'identifier les stratégies les plus à même de le gérer en proposant un plan d'intervention (Hart, Boers, 2009). Le LS/CMI (Level of Service Inventory/Case Management Inventory) a été conçu dans cette perspective par les chercheurs canadiens Andrews, Bonta et Wormith dans les années 2000 (Andrews et al., 2005). Il constitue désormais l'instrument privilégié des services correctionnels canadiens (Guay, 2013). Les dernières recherches relatives aux facteurs de récidive ont enfin contribué à mettre en exergue l'importance des facteurs dits de protection dans l'évaluation du risque de récidive. Ces facteurs positifs, internes, externes et motivationnels sont susceptibles de modérer ou de compenser la présence d'un facteur de risque (Parent et al., 2009 ; Guay 2013) en exerçant des influences qui modifient, améliorent ou changent les réactions d'une personne aux risques environnementaux qui prédisposent à une mauvaise adaptation (Rutter, 1985, 600). S'il s'agit toujours de gagner en prédictibilité, leur inclusion n'est sans doute pas étrangère à la volonté de départir les instruments d'évaluation de la connotation répressive qui leur est généralement associée, en présentant une vision moins négative et moins réductrice du justiciable. Le premier instrument exclusivement dédié à l'étude des facteurs de protection, la Structured Assessment of Protective Factors (SAPROF), a été créé par des Néerlandais (Vries Robbé et al., 2011 ; Vogel et al., 2011 ; Vries Robbé et al., 2012). Les services anglais ont également développé depuis 2006 un outil dit de quatrième génération, OASys (Offender Assessment System), qui intègre ces facteurs de protection aux côtés des facteurs statiques et dynamiques (Newbold, 2011). Aux côtés de ces outils plus ou moins scorés, d'autres outils d'aide à la décision existent, qui viennent non plus se substituer au jugement clinique mais davantage le soutenir, le structurer. Contenant une liste d'items à évaluer, sorte d'aide-mémoire à destination des professionnels, ils permettent ainsi la formulation d'un jugement professionnel structuré mais non nécessairement scoré. Bien qu'ils s'appuient à la fois sur des facteurs statiques et dynamiques, ceux-ci ne sont pas nécessairement reliés entre eux par un rapport de proportionnalité mathématique, de sorte que les évaluateurs doivent apprécier eux-mêmes le poids relatif de chaque item (Niveau, 2011 ; Guay, 2013). Certains de ces instruments sont relativement anciens, comme le Historical-Clinical-Risk (HCR-20) (Webster et al., 1997), constitué de 20 items, historiques ou statiques, cliniques et associés à la gestion du risque. Cet outil a été décliné sous plusieurs formes dans l'objectif d'évaluer toujours plus finement la récidive en matière de violence sexuelle (Sexual Violence Risk-20 (SVR-20); Boer et al., 1997), ou de violence conjugale (Spousal Assault Risk Assessment Guide (SARA); Kropp et al., 1999). Cette méthode semi-structurée est jugée prometteuse dès lors que l'évaluateur reste juge de la pondération à appliquer aux différents items et de l'évaluation finale du niveau de risque (Gravier, Lustenberg, 2005, 676).

Bien que la mobilisation de ces recherches étrangères reste encore confidentielle en France, les méthodes d'évaluation mises en œuvre par les CPIP ont progressivement été remises en cause. Les agents de probation ont été invités par l'Administration Pénitentiaire à structurer leur jugement professionnel, pour proposer des évaluations harmonisées, plus fiables, garantissant l'individualisation des prises en charge.

\section{II - La tentative de structuration des méthodes d'évaluation des justiciables : une appropriation délicate}

Face au constat de la faiblesse opérationnelle des évaluations conduites par les CPIP, des responsables politiques et institutionnels français, comme divers praticiens, ont promu non pas l'importation sans nuance des instruments actuariels, mais a minima le développement d'approches plus structurées que par le passé. C'est dans ce contexte que le Diagnostic à visée criminologique (DAVC) a été introduit au sein des SPIP à partir de 2008. En raison 
des difficultés d'appropriation rencontrées par les personnels, relayées par les principaux syndicats, l'instrument a fait l'objet d'une utilisation limitée. Sa mise en œuvre a été suspendue dès l'été 2013, avant que le Conseil d'État n'annule sa circulaire d'application en avril $2014^{10}$. $\mathrm{Si}$ le caractère central de l'évaluation des justiciables est désormais reconnu et consacré au plan européen (Conseil de l'Europe, 2006, 103.2 ; Conseil de l'Europe, 2010, 66) et national, un consensus peine toutefois à se dégager sur les outils devant être utilisés.

\section{1) L'introduction du DAVC : une volonté institutionnelle}

Les méthodes d'évaluation utilisées par les CPIP ont été questionnées en vue d'une harmonisation et d'une structuration de leurs pratiques professionnelles. La conception d'une grille d'évaluation nationale a été initiée localement et empiriquement à la fin des années 2000 (Larminat, 2013a). La Direction de l'administration pénitentiaire s'est ensuite saisie de cette démarche en constituant un groupe de travail composé de personnels d'insertion et de probation (agents de probation et cadres), mais également de responsables de l'Administration Pénitentiaire ${ }^{11}$. Le groupe de travail a conçu un nouvel outil d'évaluation : le Diagnostic à visée criminologique (DAVC), s'appuyant essentiellement sur les connaissances empiriques des professionnels. Les travaux étrangers relatifs aux facteurs de risque tout comme aux instruments existants d'évaluation n'ont été que faiblement ou implicitement mobilisés, cette démarche étant réputée affaiblir le cadre théorique initial de l'instrument. Expérimenté à partir de 2008, le DAVC a subi de nombreuses modifications de fond et de forme, donnant lieu à une quinzaine de versions successives. Selon les personnels qui ont participé au groupe de travail initial, ces modifications ont contribué à éloigner considérablement l'outil de sa conception initiale et des attentes des professionnels. Présenté au sein d'une circulaire du 8 novembre 2011, le DAVC a été généralisé à compter du $1^{\text {er }}$ mars $2012^{12}$, sans que les services ne bénéficient d'un accompagnement pédagogique adapté. Initialement accompagné d'un guide d'utilisation, qui n'a par la suite pas été diffusé au sein des services, l'outil a été intégré à l'application informatique APPI (Application des Peines, Probation et Insertion), application dont se servent les agents dans le cadre de leur suivi, laissant les personnels s'approprier seuls ce nouveau module. Afin de contourner certaines résistances, liées à l'imposition de cet outil par la voie hiérarchique, la Direction de l'Administration Pénitentiaire s'est efforcée d'en atténuer le caractère novateur. La circulaire de 2011 précisait ainsi que le DAVC [reprenait] pour l'essentiel des critères d'analyse déjà existants, mais ces derniers [s'inscrivaient] désormais dans le cadre d'une méthodologie harmonisée et partagée pour tous les SPIP sur le territoire national. Toutefois, au-delà de cette volonté d'harmonisation des pratiques, l'introduction du nouvel outil s'inscrivait dans une nouvelle logique, répondant à l'exigence de sécurisation de l'évaluation en vue d'une prévention efficace de la récidive et d'une meilleure gestion des risques.

Dans son ultime version, le DAVC se décomposait en cinq domaines successifs : la situation pénale et le respect de la mesure ou de la peine et de ses obligations ; l'appropriation de la condamnation et la reconnaissance de l'acte commis ; l'inscription dans l'environnement social, familial et les capacités au changement ; la situation médicale et sa compatibilité avec le projet d'insertion. La dernière phase du diagnostic, conçue comme étant le fruit $d u$ travail d'analyse et d'évaluation effectué par les CPIP tout au long des étapes précédentes, devait mettre en exergue les freins et les atouts au travail avec [le justiciable], et proposer des modalités de suivi adaptées au profil criminologique de la personne ${ }^{13}$. Cette évaluation initiale, prévue pour être actualisée au cours de la prise en charge, était enserrée dans des conditions temporelles précises. Elle devait démarrer dès le premier entretien et être finalisée à l'issue d'un délai contraint de trois mois en milieu ouvert (et d'un mois en milieu fermé). Les facteurs pris en compte, statiques et dynamiques, étaient évalués tant sur la base des pièces judiciaires ou médicales du dossier qu'à partir des éléments fournis par la personne suivie dans le cadre d'entretiens individuels. La nature des informations recueillies ne différait donc pas des pratiques antérieures. Contrairement aux outils actuariels, l'appréciation formulée à partir du DAVC ne donnait pas lieu à un résultat scoré, chaque item reposant sur une appréciation binaire (oui / non), ternaire (acquis / non acquis / en cours d'acquisition) ou à une 
appréciation libre mais nécessairement concise, le nombre de caractères étant limité. Les CPIP disposaient toujours d'une marge d'appréciation quant à la manière d'aborder les items, dont la définition induit des appréciations subjectives, sinon des jugements de valeur moralisateurs (Larminat, 2013a). En cela, cet outil repose toujours sur le jugement professionnel clinique du CPIP, ne remettant a priori que partiellement en cause leur méthode classique d'évaluation. Les agents s'appuyaient en effet sur les mêmes items et disposaient d'une liberté dans leur interprétation. Toutefois, leur appréciation était plus encadrée, ce qui permettait, selon les promoteurs du DAVC, une meilleure structuration de leur jugement professionnel. Ce faisant, l'outil semblait paré de nombreuses vertus, l'évaluation du justiciable constituant un véritable acte professionnel, reposant sur une méthode harmonisée au sein de l'ensemble des SPIP.

\section{2) La mise en œuvre du DAVC : des résistances professionnelles}

Le DAVC a pourtant fait l'objet d'une mise en œuvre limitée (Milburn, Jamet, 2013). Bien que de nombreux CPIP regrettent le manque d'outils professionnels dont ils disposent et des méthodes d'intervention confinant au bricolage, l'introduction du diagnostic s'est heurtée à des difficultés d'appropriation de la part des agents, sinon à un rejet quasi-unanime, nourri par la vive opposition des principaux syndicats. En proie à une crise identitaire persistante (Lhuilier, 2007) et dans une situation matérielle, humaine et financière délicate, les CPIP ne se sont pas pleinement investis, ou sentis investis, dans le processus d'élaboration du nouvel instrument d'évaluation. Le DAVC a en outre été perçu comme témoignant d'un désaveu institutionnel à l'égard des pratiques au sein des SPIP, profondément marqués par l'affaire dite de Pornic. Dans le contexte judiciaire de résurgence du concept de dangerosité (Kaluszynski, 2008 ; Danet, 2008 ; Lazerges, 2012), l'outil a pu être assimilé à un outil d'évaluation de la dangerosité ou du seul risque de récidive, témoignant de l'ancrage des missions des services dans une dimension plus sécuritaire que réhabilitatrice. L'intitulé de l'instrument est venu renforcer ces crispations identitaires. Et la faiblesse de l'accompagnement institutionnel n'a pas été de nature à rassurer les personnels. L'absence de référentiel d'utilisation et les carences des formations proposées, portant non pas tant sur les enjeux généraux de l'évaluation, mais davantage sur les aspects techniques de l'utilisation de l'instrument (ISP, 2013, 28), ont notamment été déplorées. En l'absence d'anticipation dans sa mise en œuvre, la sensibilisation des agents à l'utilisation du DAVC dans le cadre de leur formation initiale a été jugée trop tardive. Quant aux sessions de formation continue, elles n'ont pas fait l'objet d'une véritable harmonisation au niveau national, chaque service, chaque direction inter-régionale restant libre dans son offre de formation, ce qui a engendré des disparités entre les services et entre les agents (ISP, 2013, 28-29). Au sein des services étudiés, un CPIP a été chargé d'expérimenter l'outil afin d'accompagner par la suite ses collègues dans leurs pratiques. L'un de ces agents référents, particulièrement intéressé par la question de l'évaluation des justiciables, assurait également des modules de formation continue au sein de différentes directions régionales. Ce choix tenait, au moins en partie, à des considérations pragmatiques, en ce qu'il permet à l'Administration Pénitentiaire de faire l'économie de la rémunération d'intervenants extérieurs. Ces agents référents n'étaient pas toujours convaincus eux-mêmes de la pertinence du DAVC. Ils se sont alors contentés de remplir un ou deux diagnostics, à titre d'exemple, sans s'y référer par la suite dans le cadre de la prise en charge et sans toujours accompagner leurs collègues dans leurs pratiques. Cette mise en œuvre erratique n'a sans doute pas permis d'exploiter l'ensemble des ressources du nouvel instrument. Elle n'a surtout pas été le support de son appropriation par l'ensemble des personnels. Le contenu même de l'outil et sa structuration interne n'ont d'ailleurs pas séduit les personnels. Outre la crainte d'une approche actuarielle, le DAVC n'a pas été perçu comme consacrant les compétences professionnelles des personnels, mais comme un instrument chronophage et inutile.

20 Les professionnels ont dénoncé son caractère réducteur et appauvrissant pour l'analyse, les CPIP s'estimant réduits à cocher des cases sans disposer de l'espace nécessaire, ni du temps suffisant, pour développer leur analyse. Ne percevant pas l'intérêt du DAVC en terme d'amélioration de la prise en charge des justiciables, les CPIP l'ont assimilé à un simple outil de contrôle, répondant avant tout à une logique managériale. La standardisation des 
informations et l'informatisation des données autorisent en effet un contrôle subtil mais croissant des professionnels en termes de productivité et d'efficacité. L'objectif n'est plus ni de réhabiliter, ni de protéger la société, mais seulement d'attester de la performance du système organisationnel (Jendly, 2012, 256). La logique managériale dénature alors les finalités originelles de la prise en charge. Pour de nombreux CPIP, le DAVC ne présente d'ailleurs pas d'intérêt majeur, en termes d'individualisation des prises en charge, par rapport aux rapports semestriels traditionnels, présentant même une moindre pertinence en termes d'informations transmises au magistrat mandant. La question des destinataires de l'évaluation a d'ailleurs constitué un point d'achoppement. Le DAVC, intégré à l'application APPI, est accessible aux CPIP mais également aux autorités judiciaires, du parquet comme du siège. Et même si les magistrats du parquet n'ont, en dehors de certaines procédures, qu'un accès consultatif ${ }^{14}$, le partage des informations reste une question délicate, nourrissant la crainte de l'instauration d'un fichage généralisé de la population pénale (ISP, 2013, 36). Les CPIP émettent par ailleurs des doutes sur la capacité des autorités judiciaires à se saisir des informations contenues dans le DAVC, à défaut d'information ou de formation suffisantes (ISP, 2013, 40-41). La sensibilisation des magistrats n'a souvent reposé que sur des actions menées localement par les directeurs des SPIP qui, soucieux de les associer à cette évolution, ont organisé des réunions de présentation ponctuelles.

L'ensemble de ces arguments explique la mise en œuvre partielle et disparate du DAVC au sein des SPIP, puis son abandon progressif. L'utilisation de l'outil a fait l'objet d'un bilan quantitatif et qualitatif au niveau national, par l'Inspection des Services Pénitentiaires (ISP, 2013). Ce rapport, qui porte sur une vingtaine de SPIP, confirme l'utilisation restreinte et partielle de l'outil. Dans les antennes de milieu ouvert étudiées au sein de ce rapport, seuls $13,4 \%$ des mesures prises en charge ont donné lieu à la réalisation du diagnostic entre mars 2012 et août 2013, avec néanmoins de fortes disparités régionales (ISP, 2013, 19-20). Au sein de nos trois services étudiés, seul un ou deux DAVC ont été réalisés sans que son utilisation ne soit ensuite généralisée, ces services illustrant ainsi sa mise en œuvre marginale. Alors que la plupart des agents se disent réticents à l'introduction d'outils actuariels, le rapport de l'ISP montre qu'au sein des DAVC validés, les champs structurés étaient plus fréquemment complétés que les champs libres (ISP, 2013, 21). Les délais de réalisation du DAVC, également critiqués, étaient paradoxalement brefs : $93 \%$ des DAVC ont été validés dans un délai inférieur au délai réglementaire de trois mois, et $79 \%$ ont même été conduits dans un délai inférieur à un mois (ISP, 2013, 23). Les perspectives d'évolution des méthodes d'évaluation des CPIP sont à l'heure actuelle incertaines, des voix divergentes s'élevant au sein du ministère de la Justice, de l'Administration Pénitentiaire, des professionnels comme des universitaires pour promouvoir ou rejeter l'introduction d'un outil actuariel. Plusieurs issues s'offrent en effet aux SPIP français entre la transposition d'outils étrangers actuariels ou semi-actuariels, de seconde, troisième ou quatrième génération, ou une meilleure structuration du jugement professionnel des CPIP.

\section{3) Les perspectives d'évolution : entre prudence et promotion des instruments actuariels}

22 Malgré les réserves de nombre de professionnels français, les instruments actuariels séduisent certains responsables politiques et institutionnels français. À la suite du rapport de JeanFrançois Burgelin, Santé, justice et dangerosité, pour une meilleure prise en charge de la récidive, qui recommandait en 2005 le développement de méthodes adaptées à l'évaluation du risque de récidive, deux rapports, celui du député Jean-Paul Garraud en 2006 puis celui du premier Président de la Cour de cassation, Vincent Lamanda, ont déploré que les praticiens français ne fassent pas plus référence aux instruments actuariels ou semiactuariels pour apprécier la dangerosité, ne serait-ce que pour soutenir le jugement clinique (Burgelin, 2005 ; Garraud, 2006 ; Lamanda, 2008). L’Académie de médecine, tout en reconnaissant l'intérêt prédictif moyen des instruments scorés, considère qu'ils devraient soutenir le jugement clinique, ne serait-ce que pour améliorer le niveau de transparence et d'homogénéité des évaluations $(2012,11)$. Le Conseil de l'Europe s'inscrit également dans 
cette voie médiane, recommandant que l'appréciation professionnelle des agents soit étayée par des instruments d'évaluation (Conseil de l'Europe, 2010, 66 et 71 ; Conseil de l'Europe, 2014, 26 à 31). Dans sa récente recommandation relative aux délinquants dangereux, il estime que l'évaluation du risque devrait être structurée, fondée sur des preuves et reposer sur des outils validés appropriés ainsi que sur un processus professionnel de prise de décision (Conseil de l'Europe, 2014, 28). Moins réservés, certains rapports nationaux prônent le recours exclusif aux outils actuariels. Dans son rapport publié pour le compte de l'Institut pour la Justice, le psychiatre Alexandre Baratta, loin des approches nuancées de la très grande majorité de ses confrères, préconise la réalisation obligatoire d'évaluations actuarielles du risque de dangerosité préalablement à l'octroi des mesures de libération conditionnelle notamment $(2011,25)$. Toutefois, la plupart des rapports publiés sur le sujet, qu'ils émanent de parlementaires, de hauts magistrats ou de praticiens demeurent plus prudents et privilégient la voie médiane, sous la forme d'évaluations semi-actuarielles ou simplement structurées. La Haute Autorité de Santé considère que les échelles actuarielles, qui s'appuient essentiellement sur des facteurs statiques pour évaluer notamment le risque de récidive violente, ne sont pas adaptées à l'évaluation psychiatrique $(2011,17)$. Le rapport de 2012 définissant les objectifs de la politique d'exécution des peines a quant à lui développé une position quelque peu confuse, préconisant, dans le cadre de l'évaluation criminologique de la dangerosité, d'intégrer les outils actuariels aux méthodes des professionnels français afin de leur permettre d'émettre des avis circonstanciés, fondés sur des critères précis ${ }^{15}$. Interpellé sur cette question, le jury de la Conférence de Consensus sur la prévention de la récidive, particulièrement prudent sur ce point, s'est contenté de réclamer des études relatives aux instruments étrangers, sans se prononcer sur des outils particuliers et en appelant à une évaluation raisonnée (2013, 35). L'étude d'impact du projet de loi relatif à la prévention de la récidive et à l'individualisation des peines, qui découle des travaux de cette Conférence, envisage de bâtir avec les professionnels concernés et en lien avec les scientifiques ayant contribué au développement d'outils perfectionnés des nouveaux instruments mieux adaptés aux besoins et à l'état des connaissances ${ }^{16}$.

Malgré cette relative prudence, les partisans des approches actuarielles se livrent à un véritable lobbying promotionnel. Selon eux, le manque d'ouverture aux acquis de la science générerait non seulement des pratiques inefficaces sinon contre-productives, mais serait par ailleurs révélateur d'un véritable manque d'éthique. Témoin d'un regrettable aveuglement idéologique, le rejet des outils actuariels serait en effet hautement liberticide, pouvant conduire, sur la base d'une évaluation hasardeuse, à une surévaluation du risque de récidive, privant les justiciables du bénéfice de mesures de libération conditionnelle, voire justifiant le prononcé de mesures de sûreté (Herzog-Evans, 2012a). Maniant parfois le registre de la culpabilisation, certains chercheurs pointent ainsi le risque pour les professionnels d'être considérés comme négligents, voire poursuivis pour faute (Douglas et al., 1999 ; Vogelvang, Tigges, 2012). Face à l'injonction qui leur est faite de protéger la société, de prédire et de traiter la délinquance, les intervenants ont effectivement une responsabilité de plus en plus lourde, illustrée par la mise en cause des personnels d'insertion et de probation suite à l'affaire de Pornic. Dans une logique immunitaire (Kaminski, 2009, 103) compréhensible, les agents de probation risquent dès lors d'utiliser les échelles actuarielles comme une assurancedysfonctionnement (Jendly, 2012, 252), permettant de justifier et de sécuriser leurs pratiques (Vacheret, 2010). Il n'est pas improbable non plus que, dans un contexte parfois concurrentiel entre JAP et agents de probation, experts psychiatres et psychologues, agents de probation et professionnels des associations socio-judiciaires, ces instruments soient mobilisés dans des enjeux de pouvoir, permettant aux uns ou aux autres de garantir leurs positions de spécialistes, sous couvert de vernis scientifique. Michel Foucault avait déjà démontré que si le crime est devenu pour les psychiatres un enjeu important au XIX ${ }^{\mathrm{e}}$ siècle, c'est [parce] qu'il s'agissait moins d'un domaine de connaissance à conquérir que d'une modalité de pouvoir à garantir et à justifier (Foucault, 1981, 409; Larminat, 2013b).

Du point de vue des politiques, l'appel à la raison scientifique permet également de légitimer leurs discours autrement que par des considérations plus ou moins morales. Ces instruments 
alimentent en effet le mythe de la rationalité des décisions publiques. Comme le souligne Robert Castel, les idéologies modernes de la prévention sont surplombées par une grande rêverie technocratique, rationalisatrice, du contrôle absolu de l'accident conçu comme irruption de l'imprévu (Castel, 1983, 123). Plus encore, les outils actuariels, en se focalisant non pas tant sur les causes de la délinquance que sur les marqueurs de risque qui les prédiraient le mieux, tendent à dépolitiser le processus de régulation sociale (Silver, Miller, 2002, 144). L'approche actuarielle contente ainsi les nouvelles exigences managériales et néolibérales fondées sur un usage optimal des ressources pénales (ibid.). Cette méthode est placée au service d'une politique de neutralisation sélective, qui concentrerait les ressources sur les délinquants présentant les plus forts risques de récidive (Harcourt, 2011a, 20). Désormais soumise aux impératifs managériaux, induisant une exigence de performance, d'efficience et d'efficacité, l'Administration Pénitentiaire se montre sensible à ces arguments (Froment, Kaluszynski, 2011 ; Péchillon, 2011), au risque de sous-estimer les limites inhérentes à l'utilisation des outils actuariels.

\section{III - Les risques inhérents à un recours accru aux outils actuariels : une prudence nécessaire}

Il est certain que les professionnels français ont à apprendre des pratiques et des recherches étrangères. Une lecture approfondie de la littérature scientifique existante dévoile toutefois, derrière le caractère $a$ priori séduisant et sécurisant des instruments actuariels, de nombreuses limites, tant en termes d'évaluation du risque que de prises en charge des condamnés. Les méthodes actuarielles, telles qu'elles sont parfois présentées par leurs promoteurs, semblent proposer une prédiction rapide, fiable et nécessairement objective. Or, cette conception réductrice occulte tout un pan de la sociologie critique (Larminat, 2013b, 57). La conception même des instruments actuariels souffre de biais méthodologiques, induisant des risques de dérives en termes d'éthique et une moindre individualisation des prises en charge des justiciables.

\section{1) Une fiabilité prédictive discutée}

Si les concepteurs des méthodes actuarielles et semi-actuarielles s'accordent pour rejeter les évaluations cliniques non structurées (Aegisdottir et al., 2006 ; Hanson et al., 2009 ), ceux-ci sont loin de s'entendre sur les mérites respectifs des échelles actuarielles «pures » et des jugements cliniques structurés (Abbott, 2011). Les partisans des outils de troisième et quatrième générations ne cessent d'ailleurs de mettre en garde contre l'utilisation abusive des méthodes centrées sur les seules variables statiques, en raison de l'absence de prise en compte de nombreux facteurs de récidive et des marges d'erreur excessives qu'elles génèrent. Campbell a notamment démontré qu'au seuil fixé pour la Static-99, $51 \%$ des auteurs d'agression sexuelle condamnés seraient libérés alors qu'ils devraient $a$ priori récidiver, tandis que $31 \%$ seraient classés à tort récidivistes (Campbell, 2003). À l'inverse, les jugements professionnels structurés, combinant évaluation actuarielle et clinique, permettraient une appréciation individuelle beaucoup plus juste, tenant compte des facteurs d'évolution de l'état clinique et de gestion future du risque (Douglas, Skeem, 2005 ; Guay, 2006 ; Cortoni, Lafortune, 2009 ; Mossman, 2013). Les défenseurs des échelles actuarielles statiques considèrent quant à eux qu'elles présentent une validité prédictive systématiquement supérieure, alors que l'inclusion de facteurs dynamiques induirait une perte de rigueur et de précision en réinjectant les difficultés posées par le jugement clinique (Krauss, Scurich, 2013, 223 ; Boccaccini, et al., 20093 ; Abbott, 20113 ; Quinsey et al., 1998). Selon Martine HerzogEvans, cette introduction d'éléments cliniques discrétionnaires a été faite afin de contourner la résistance psychologique des praticiens (agents de probation, forensic psychologists...) face aux outils actuariels. En réalité, elle n'ajoute strictement rien à la qualité de l'évaluation qui est faite et présente même le risque, en introduisant de l'appréciation discrétionnaire, d'en réduire la fiabilité [...] Comme le disent Quinsey et alii : Les mesures actuarielles sont trop bonnes et les jugements cliniques trop faibles pour que l'on puisse se permettre de prendre le 
risque de contaminer le premier par le second (2012a). D'autres encore considèrent qu'il n'y a pas de différences marquées en termes de validité prédictive (Singh, Grann, Fazel, 2011). L'introduction de variables dynamiques pose effectivement de lourdes difficultés d'un point de vue méthodologique, dès lors qu'elles supposent une analyse de processus sur la base d'indicateurs relativement instables et interdépendants (Campbell, DeClue, 2010). Les items retenus sont extrêmement difficiles à coder dans une grille statistique, surtout dans des échelles qui demeurent bien souvent dichotomiques (oui/non ; présent/absent) et donc réductrices. Les items retenus reposent en outre sur des appréciations amplement subjectives, comme le montrent les variables contenues dans différents types d'outils : «style de vie antisociale », «peu de remords/blâme la victime », nombre de pairs « positifs », « manque de coopération avec l'agent de probation », « manque d'hygiène », «pourrait mieux gérer son temps », « relations peu gratifiantes avec ses parents », « soins parentaux déficients », «pauvres habiletés sociales ", " faible performance », « supervision inadéquate », " problèmes avec ses professeurs », etc. L'enregistrement de telles variables est loin d'être un exercice statistiquement neutre (Hannah-Moffat, 2013b, 295). Outre qu'il est peu probable qu'ils soient interprétés de manière uniforme, ces critères peuvent impliquer beaucoup de spéculation et de jugements moraux subjectifs (Hannah-Moffat, Maurutto, 2003, 14). Ces méthodes réintroduisent le pouvoir discrétionnaire des évaluateurs, de sorte que l'on en revient au bricolage reproché jusqu'alors aux cliniciens. Certes, leurs promoteurs répondent que les outils tels que le LSI-R limitent au maximum les possibilités d'ajustement clinique (moins de 10\% pour le LSI-R), les évaluateurs étant par ailleurs obligés de motiver leur décision. Faute de temps et dans un contexte de responsabilité accrue, les agents seraient peu enclins à s'éloigner des scores obtenus (Jendly, 2012, 247). Toutefois, les professionnels chargés de l'évaluation auraient alors tendance à modifier, sans y être autorisés, le score de telle ou telle variable, cette pratique totalement invisible excluant tout rapport motivé (Hannah-Moffat, 2013b, 285). Il n’y a pas davantage de consensus concernant les points de coupure permettant d'établir le degré du risque de récidive au sein des outils scorés (Millaud, Dubreucq, 2012, 433) ou les prédicteurs de récidive à prendre en compte, comme en attestent les débats autour de la pertinence de l'inclusion du déni (Nunes et al., 2007 ; Lund, 2000).

La plupart des outils d'évaluation, y compris les mieux évalués, présentent une validité prédictive modérée ou moyenne (Millaud, Dubreucq, 2012 ; Voyer, Senon, 2012 ; Guay, 2013). Alors même que les facteurs de risque se recoupent généralement dans les différents instruments d'évaluation, il n'est pas rare qu'ils aboutissent à des résultats divergents (Mills, Kroner, 2006 ; Vrieze, Grove, 2010). Barbaree, Langton et Peacock (2006) ont constaté que moins de $8 \%$ des délinquants sexuels sont constamment déclarés délinquants à risque élevé ou à faible risque par cinq échelles actuarielles couramment utilisées (VRAG, SORAG, Static-99, ERRRS et MnSOST-R) (voir également Krauss, Scurich, 2013). Dans de nombreuses hypothèses, la fiabilité des outils d'évaluation est même surévaluée. Les chercheurs n'étant pas plus infaillibles que les praticiens, leur désir conscient ou inconscient de produire des résultats statistiquement significatifs, renforcé par les pratiques éditoriales et commerciales de certaines revues scientifiques nord-américaines (McSherry, 2013) soutenant les outils actuariels, n'est pas sans incidence sur leurs résultats (Simmons et al., 2011, 1-2). À cet égard, le fait qu'un nombre important d'évaluations des instruments actuariels ou semi-actuariels soient réalisées par leurs propres concepteurs n'est pas sans susciter des interrogations (Singh et al., 2013). Il n'est pas rare que ces auteurs concluent à une prédictivité accrue de l'outil par rapport aux résultats issus des recherches indépendantes (Blair et al., 2008 ; Singh et al., 2013). Au-delà des enjeux de reconnaissance sur la scène scientifique, les considérations financières sont en outre loin d'être anodines, car ces échelles, les manuels et les formations qui les accompagnent sont commercialisés, K. Hannah-Moffat (2013a, 131) évoquant même une véritable riskassessment industry.

\section{2) Des biais méthodologiques sous-estimés}

Au-delà du contenu même des outils, ce sont également les conditions méthodologiques de leur élaboration, et principalement la composition des cohortes de référence qui posent 
problème. A l'instar de récentes recherches qualitatives (Singh et al., 2014), Dominique Deprins rappelle les problèmes bien connus de choix du modèle, de choix des variables, de choix des plans d'échantillonnage, de l'écart entre ce que l'on veut mesurer et ce que l'on mesure effectivement, de l'ignorance des balises à l'interprétation et des mesures de fiabilité ou d'incertitude qui accompagnent toute estimation d'un modèle probabiliste (on "probabilise » l'erreur), du non-respect des hypothèses sous-jacentes aux modèles, etc. (Deprins, 2006, 509). Au titre des biais d'échantillonnage, les groupes retenus dans les études pour construire les instruments actuariels ne sont pas nécessairement représentatifs de la population dans laquelle se trouve l'individu à évaluer (Niveau, 2011). Ainsi, le VRAG et la Static-99 ont été élaborés ou testés sur des échantillons de personnes de sexe masculin, enfermées dans des établissements hospitaliers ou des établissements pénitentiaires de haute sécurité ou de sécurité maximale. Le taux de base en matière de récidive pourrait dès lors être plus bas parmi l'ensemble des délinquants que les taux calculés par les échelles actuarielles (Hannah-Moffat, $2013 \mathrm{a}, \mathrm{b}$ ). Certains échantillons sont parfois modestes, et même très modestes (moins de 100 personnes), ce qui limite de fait la validité statistique des études engagées (Hart et al., 2007, 2013). De surcroît, les délinquants concernés sont principalement nord-américains, et notamment canadiens. La délinquance étant indissociable du contexte national, politique, économique et culturel dans lequel elle se manifeste, il serait fort hasardeux de transposer ces échelles sans procéder à des études de cohortes nationales préalables.

Ces échelles ont par ailleurs été construites sur des échantillons composés d'hommes blancs, de sorte que leur capacité prédictive concernant les femmes et les minorités est loin d'être assurée (Hannah-Moffat, 2013 a ; Morash, 2009 ; Van Voorhis et al., 2010 ; Singh et al., 2011). D'autres biais de sélection sont liés au processus judiciaire lui-même. Les données les plus fréquemment collectées pour analyser les risques de récidive proviennent de sources d'information officielles, qui ne reflètent en rien la réalité de la délinquance ou des phénomènes de réitération mais constituent au moins partiellement une construction sociale. La question se pose notamment pour les condamnés pour agression sexuelle, qui ne reflètent pas nécessairement l'ensemble des délinquants sexuels dès lors qu'une minorité seulement d'infractions est suivie d'une plainte et a fortiori d'une condamnation (Vrieze, Grove, 2010 ; ONDRP, 2012, 101). Le nombre d'antécédents, qui constitue la variable la plus influente dans les échelles actuarielles comme dans les jugements professionnels structurés, dépend également des évolutions législatives et des pratiques judiciaires. Pour ce qui concerne la France, la progression du taux de réponse pénale (plus de vingt points en quinze ans) et la modification des orientations procédurales ont engendré une croissance artificielle du nombre de réitérants et de récidivistes au sens légal du terme (Gautron, 2014). Si ces outils d'évaluation étaient d'usage courant, les délinquants français seraient dès lors considérés comme présentant davantage de risques de récidive au fil du temps, quand bien même leur niveau d'activité délinquante ne changerait pas.

Ces échelles induisent également de véritables discriminations, car le passé pénal est inextricablement lié à l'emploi, la race, l'âge, le genre (Bushway, Morrison Pielh, 2007 ; Crow, 2008 ; Harcourt, 2010, Hannah-Moffat, 2013 a, b ; Starr, 2014). En France comme à l'étranger, les minorités sont plus fréquemment contrôlées par la police et plus lourdement condamnées (Chiricos et al., 2004 ; Jobard, Névanen, 2007 ; Pager, 2008 ; Jobard et al., 2009 ; Harcourt, 2010 ; Crutchfield et al., 2012 ; Muller, Widleman, 2013). Dans une recherche sur l'évolution des modes de traitement des délits dans cinq juridictions du Grand Ouest français, Virginie Gautron et Jean-Noël Retière ont ainsi démontré que toutes choses égales par ailleurs, les personnes nées à l'étranger voyaient multiplier par trois la probabilité d'une comparution immédiate, cette procédure multipliant par huit la probabilité d'une peine d'emprisonnement ferme (Gautron, Retière, 2013). La probabilité d'une détention provisoire était quant à elle multipliée par cinq, cette incarcération préalable multipliant également par huit le risque d'un emprisonnement ferme. À l'identique, les personnes sans emploi et les sans domicile fixe apparaissaient plus défavorablement traitées par le système pénal. Dans l'hypothèse où ces outils prendraient davantage de place dans le processus pénal français, les populations les plus marginalisées seraient, comme à l'étranger, systématiquement jugées 
« plus à risque» (Hudson, Bramhall, 2005 ; Martel et al., 2011 ; Raynor, Lewis, 2011 ; Oleson, 2011 ; Harcourt, 2011b ; Hannah Moffat, 2013 a, b). En raison de l'exposition plus élevée des groupes marginalisés au risque, à la discrimination raciale, aux inégalités sociales, l'inclusion des facteurs dynamiques pourrait contribuer à accentuer encore ce processus discriminatoire (Hannah-Moffat, 2013 b, 281-282). Ces facteurs reproduisent des formes de discrimination systémique, comme le montre l'exemple du critère relatif à la stabilité professionnelle, évaluée au fil des prises en charge. En effet, des études ont mis en évidence l'étendue différenciée du désavantage associé à un casier judiciaire en matière d'accès à l'emploi, au détriment des minorités et plus particulièrement des afro-américains (Pager, 2003 ; Wakefield, Uggen, 2010). En 2003, la Commission canadienne des droits de l'homme (CHRC) a d'ailleurs critiqué l'emploi et le contenu des variables dynamiques, ces facteurs de risque étant liés à des motifs de discrimination interdits (Hannah-Moffat, $2013 \mathrm{a}, \mathrm{b}$ ). Les variables de risque statiques comme dynamiques ne peuvent être extraites du contexte socio-politique, économique et culturel (Hannah-Moffat, 2013 a, b ; Silver, Miller, 2002). Or, une fois considérés comme étant plus à risque, ces publics, socio-économiquement fragiles, feront l'objet d'un suivi plus intensif, augmentant mécaniquement la probabilité d'éventuels manquements aux modalités de leur suivi (Hannah-Moffat, 2013 a, b). Les échelles actuarielles contribuent ainsi à la marginalisation des populations déjà en marge de la vie politique et économique (Silver, Miller, 2002).

\section{3) Une individualisation des prises en charge menacée}

La détermination des méthodes d'évaluation utilisées par les professionnels français mérite une réflexion approfondie, tenant compte des limites évoquées. En effet, une fois élaborés, les instruments ont vocation à servir de fondement à l'évaluation des justiciables, conditionnant la nature de la prise en charge ou les modalités d'exécution de la peine. Or, ils comportent des risques de dérive du point de vue des libertés individuelles.

Au regard de la fiabilité incertaine des instruments évoqués, le recours aux outils actuariels comporte le risque de donner lieu à une évaluation inexacte de la probabilité de récidive (Vacheret et al., 1998 ; Freedman, 2001 ; Szmukler, 2001 ; Farrington et al., 2008). D’un point de vue éthique, l'importance des taux de faux positifs et de faux négatifs exige une réelle prudence vis-à-vis des évaluations standardisées (Buchanan, 2008, 184 ; Padfield, 2010). Il suffit de citer le calcul effectué en 1968 par Livermore et ses collaborateurs pour se convaincre de leur potentiel hautement liberticide : admettons, écrivent-ils, qu'une personne sur mille va tuer et admettons qu'un test très précis puisse différencier avec 95\% d'efficacité ceux qui vont tuer de ceux qui ne vont pas tuer. Si nous testions 100 000, sur les 100 qui vont tuer, 95\% seraient retenus. Mais malheureusement, sur les 99900 qui ne vont pas tuer, 4995 personnes seraient retenues comme des tueurs potentiels (cité in Archer, 2007). Il existe dès lors une première forme de dangerosité qui consiste à s'attaquer aux droits des individus en les agressant physiquement, il en existe une seconde qui consiste aussi, en se prononçant sur la dangerosité des autres, à faire violence à leurs droits fondamentaux. Il n'est pas évident que l'une soit plus excusable que l'autre (Dozois et al., 1981, 398). De récentes études en appellent ainsi à la vigilance concernant l'utilisation judiciaire des estimations probabilistes (Singh et al., 2014). Bien que peu de recherches aient été conduites pour mesurer leur impact sur les pratiques décisionnelles, il est à craindre que les évaluations scorées viennent supplanter d'autres critères susceptibles d'orienter le choix de la peine, voire de la condamnation (Hannah-Moffat, 2013 b ; Reichman, 1986 ; Starr, 2014). Ces risques sont d'autant plus importants que les praticiens ne sont pas familiers des calculs probabilistes. Peu interprètent correctement les scores de probabilité, avec des confusions fréquentes entre corrélation et causalité (Hannah-Moffat, 2013 b). Au regard de l'influence des outils scorés, certains experts européens ont renoncé à ce jour, à présenter des résultats chiffrés du risque de récidive, d'une part, en raison des faiblesses méthodologiques [...] et, d'autre part, en raison de l'impact qu'ont les données chiffrées dans une cour de justice. Ces données, qui devraient être manipulées avec la plus grande prudence, ont habituellement pour effet d'écraser toute 
possibilité de nuance, en raison de l'apparence de scientificité que porte ce type d'information par rapport au verbe (Delacrausaz, Gasser, 2012, 442).

Dès lors que l'évaluation peut conclure à un risque faible de récidive au sujet d'individus qui en réalité récidiveront (faux négatif), les outils actuariels n'exposent pas moins la société à de graves dangers. À l'inverse, un faux positif (une personne jugée à risque qui ne récidivera pas) se traduira par une prise en charge renforcée et plus contraignante pourtant inutile. Ces outils ne contentent donc pas davantage les exigences du nouveau management public en termes de rationalisation des moyens humains et financiers. En niant les spécificités individuelles des justiciables, le processus d'individualisation des peines et de leur exécution s'en trouve également gravement affecté (Hannah-Moffat, 2013 b, 277). Le classement des délinquants selon leur niveau de risque de récidive ne s'intègre plus dans une perspective de réintégration sociale. Il permet davantage de mettre en place un système de contrôle social gradué afin de contenir les problématiques individuelles plutôt que de les résoudre (Silver, Miller, 2002). La Nouvelle Pénologie, dont relèvent les outils actuariels, recherche principalement la protection de la société en gérant de manière rationnelle et objective des groupes considérés à risque (Slingeneyer, 2007 ; Mary, 2001 ; Robinson, 2002). Si l'individualisation a originellement guidé la détermination des premiers facteurs de risque, il s'agit désormais d'éviter la commission d'une nouvelle infraction en optimisant l'allocation des ressources pénales en fonction du niveau de risque. Dans le cadre de ce processus de rationalisation, les modalités du suivi des délinquants sont appelées à être standardisées, uniformisées en fonction du profil du délinquant. Il s'agit ainsi non plus de proposer des modalités de suivi adaptées à la personnalité du délinquant et à sa situation, mais de proposer un suivi rationnel, efficient et effectif (Chantraine, Cauchie, 2006). En fonction du niveau de risque de récidive, les praticiens doivent intégrer le délinquant dans l'une des typologies de suivi préalablement déterminées. Or, les risques identifiés, définis comme des besoins criminogènes auxquels la prise en charge devra répondre, restent des facteurs généraux, déterminés statistiquement pour un groupe de délinquants, ne correspondant pas nécessairement aux besoins réels de chaque délinquant. Cette évolution est d'ailleurs perceptible au sein des SPIP, au regard de l'instauration d'une nouvelle typologie de prise en charge, reposant sur cinq segments qui correspondent à des groupes de justiciables présentant des problématiques communes mais également un profil similaire en termes de quantum, de nature de peine et de perspective d'évolution (Ministère de la Justice, 2011).

Le prisme par lequel le justiciable est appréhendé nuit à l'instauration d'une véritable relation interpersonnelle avec les agents de probation. Le temps passé à réaliser ces évaluations réduit d'autant celui consacré au suivi et à l'accompagnement (Milburn, Jamet, 2013). Depuis la mise en place d'OASys, en Grande-Bretagne, l'un des formateurs à cet instrument reconnaît que les agents de probation anglais consacrent $75 \%$ de leur temps à des tâches qui n'impliquent pas de contact avec les auteurs d'infractions (Forbes, 2012). L'optimisation des outils d'évaluation suppose ainsi l'intervention d'un personnel compétent, formé et donc possiblement différent de celui chargé du suivi, ce qui induit une spécialisation des agents. Au plan national, les SPIP sont invités à repenser leur mode d'organisation, le principe de la polyvalence des agents étant progressivement supplanté par celui de leur spécialisation. Dans le contexte actuel de révision générale des politiques publiques (RGPP), les SPIP sont appelés à être réorganisés par pôles d'activité, autour notamment d'un «pôle enquête » et d'un «pôle suivi ». Ce nouveau principe d'organisation est progressivement mis en place dans les services. Cette réorganisation induit une fragmentation de la prise en charge, qui se traduit par la multiplication des interventions ponctuelles et ciblées au détriment d'une prise en charge globale et continue par un seul CPIP référent (Larminat, 2014 a, b). La spécialisation renforce le mouvement de déresponsabilisation des personnels, en opérant une dilution des responsabilités sur l'ensemble des intervenants (Quirion et al., 2012). Dans une perspective de gestion des risques et de régulation des déviances, le souci de contrôle du délinquant et de protection de la société justifie enfin le développement d'un continuum pénal, c'est-à-dire de mesures qui viennent prolonger la surveillance en dehors des murs, de manière diffuse et continue (Slingeneyer, 2007 ; Larminat, 2014 dans ce dossier). En parallèle de contrôles 
accrus en milieu libre, cette logique prudentielle pourrait se traduire par une décroissance du nombre d'aménagements de peine, comme le confirme la diminution du nombre de libérations conditionnelles au Canada depuis l'introduction des outils actuariels (Vacheret, Cousineau, 2005). L'efficacité des mesures du milieu ouvert en termes de diminution du taux récidive est pourtant fréquemment soulignée, bien que ces résultats doivent être lus en intégrant le processus de sélection judiciaire des justiciables bénéficiant de ces mesures (Kensey, Benadoua, 2011).

\section{Conclusion}

La question des méthodes d'évaluation des justiciables revêt une importance capitale dans leur prise en charge. S'il s'avère indispensable de doter les conseillers pénitentiaires d'insertion et de probation (CPIP) de nouveaux instruments leur permettant d'affiner leur jugement professionnel, il faut garder à l'esprit que la prise en charge des délinquants constitue toujours un pari sur l'humain et qu'il est impossible de prédire avec certitude et infaillibilité le risque de récidive d'un individu. Si l'élaboration d'un nouvel instrument d'évaluation comporte des enjeux essentiels en termes de qualité et d'efficacité de la prise en charge des justiciables, ce n'est qu'à la condition de lui assigner une finalité plus ambitieuse que la simple détermination d'un niveau de risque de récidive. L'intervention auprès des justiciables ne doit pas se réduire au seul objectif de prévenir la récidive mais s'inscrire dans une perspective de réhabilitation, de sortie de la délinquance ou désistance afin de permettre aux justiciables de réintégrer la société, qui n'en sera que mieux protégée. Renouant avec le principe de l'individualisation, les missions des SPIP s'en trouveraient ainsi valorisées, laissant entrevoir une clarification de l'identité professionnelle des agents. Pour l'heure, l'Administration Pénitentiaire poursuit ses réflexions en vue d'une meilleure structuration du jugement professionnel des CPIP. Au regard de l'échec de la mise en œuvre du DAVC, un nouveau groupe de travail a été mis en place en septembre 2013 afin d'élaborer un nouvel instrument d'évaluation des risques de récidive des justiciables français, anticipant l'annulation de la circulaire d'application par le Conseil d'État en avril 2014. Mais sa composition ne fait pas l'unanimité en termes de représentativité des différents courants idéologiques s'opposant sur la délicate question de l'évaluation. $\mathrm{Au}$ soutien de ses réflexions, l'Administration Pénitentiaire a lancé un appel d'offre, en juin 2014, invitant des chercheurs à se pencher sur l'évaluation initiale des justiciables en accompagnant l'expérimentation de nouveaux outils standardisés au sein des SPIP.

Nous avons tenté, dans le cadre de cet article, de démontrer qu'il n'y a pas, et qu'il n'y aura jamais, d'instrument totalement fiable pour évaluer les risques de récidive. Loin d'un constructivisme radical qui rejetterait en bloc les enseignements des études américaines et canadiennes, nous ne considérons pas pour autant que les pratiques françaises n'auraient rien à apprendre des approches étrangères. Comme l'indique un psychiatre, une évaluation exclusivement centrée sur l'intuition clinique peut être aussi redoutable tant elle peut s'obnubiler de la violence du sujet et de la peur qu'il suscite (Gravier, cité in Moulin et al., 2012, 625), surtout lorsque l'expert se contente d'extraire tel ou tel élément d'observation pour en déduire toutes sortes de raisonnements, sans expliquer ni les motivations de ses choix, ni les fondements théoriques sur lesquels il les base (Delacrausaz, Gasser, 2012, 441). Nous ne rejetons pas davantage les méthodes scientifiques quantitatives qui font cruellement défaut en France, principalement en raison du manque de financements consacrés à la recherche publique et parce que nos responsables politiques sont étonnamment plus enclins à réclamer l'évaluation du risque individuel de récidive que celle des résultats de leurs politiques. Il convient toutefois d'aborder la question des méthodes d'évaluation des justiciables avec prudence en se gardant de passer d'une position réfutant par avance et par principe toute pertinence aux tentatives pronostiques, à une attitude rigidement pseudoscientifique, arcboutée sur des données statistiques (Delacrausaz, Gasser, 2012, 441). Si les outils actuariels et semi-actuariels permettent au clinicien d'intégrer à sa pratique les données de la recherche et de sélectionner l'information la plus pertinente pour une évaluation rigoureuse du risque (Millaud, Dubreucq, 2012, 435), nous souhaitons, en tant que chercheurs, mettre en garde contre une standardisation excessive et scorée de l'évaluation des risques de récidive et 
rappeler que le jugement professionnel, aussi artisanal qu'il soit, n'en demeure pas moins essentiel.

\section{Bibliographie}

Abbott B. R., 2011, Throwing the baby out with the bath water: is it time for clinical judgment to supplement actuarial risk assessment?, The Journal of the American Academy of Psychiatry and the Law, $39,2,222-230$.

Académie Nationale de Médecine, 2012, Évaluation de la dangerosité psychiatrique et criminologique, Rapport.

Aegisdottir, S., White, M. J., Spengler, P. M., Maugherman, A. S., Anderson, L. A., Cook, R. S., 2006, The meta-analysis of clinical judgement project: Fifty-six years of accumulated research on clinical versus statistical prediction. The Counselling Psychologist, 34, 3, 341-382.

Andrews D. A., Bonta A., 2007, Modèle d'évaluation et de réadaptation des délinquants fondé sur les principes du risque, des besoins et de la réceptivité, Sécurité Publique du Canada.

Andrews D. A., Bonta J., Wormith J. S., 2005, Level of Service / Case Management Inventory, Profile report, Sécurité Publique du Canada.

Archer E., 2007, Difficultés et limites de l'expertise psychiatrique de pré-libération. Les questions posées à l'expert, in Haute Autorité de Santé, Audition publique sur l'expertise psychiatrique pénale, Texte des experts.

Archer R. P., Buffington-Vollum J. K., Stredny R. V., Handel R. W., 2006, A survey of psychological test use patterns among forensic psychologists, Journal of Personality Assessment, 87, 84-94.

Baratta A., 2011, Évaluation et prise en charge des délinquants et criminels sexuels, Paris, Institut pour la justice.

Barbaree H. E., Langton C. M., Peacock E. J., 2006, Different actuarial risk measures produce different risk rankings for sexual offenders, Sexual Abuse: A Journal of Research and Treatment, 18, 423-440.

Benbouriche M., Ventéjoux A., Lebougault M., Hirschelmann A., 2012, L'évaluation du risque de récidive en France : expériences et attitudes des conseillers pénitentiaires d'insertion et de probation, Revue internationale de criminologie et de police technique et scientifique, 65, 3, 305-318.

Bernat de Celis J., 1980, L'expérience des enquêtes sociales rapides, Revue de science criminelle et de droit pénal comparé, 4, 957-967.

Blair P. R., Marcus D. K., Boccaccini M. T., 2008, Is there an allegiance effect for assessment instruments? Actuarial risk assessment as an exemplar, Clinical Psychology: Science and Practice, 15, $4,346-360$.

Blanc E., 2007, Rapport d'information $n^{\circ} 505$ sur l'exécution des décisions de justice pénale concernant les personnes majeures, Paris, Assemblée nationale.

Blanc E., 2011, Rapport d'information $n^{\circ} 4421$ sur le suivi des auteurs d'infractions à caractère sexuel, Paris, Assemblée nationale.

Boccaccini M. T., Murrie D. C., Caperton J. D., Hawes S., 2009, Field validity of the static-99 and MnSOST-R among sex offenders evaluated for civil commitment as sexually violent predator, Psychology, Public Policy and the Law, 15, 278-314.

Boer D. P., Hart S. D., Kropp P. R., \& Webster, C. D., 1997, Manual for the Sexual Violence Risk-20: Professional guidelines for assessing risk of sexual violence, Vancouver, $\mathrm{BC}$, The British Columbia Institute Against Family Violence.

Bonta J., 1997, La réadaptation des délinquants, de la théorie à la pratique, Travaux publics et services gouvernementaux, Canada.

Buchanan A., 2008, Risk of Violence by Psychiatric Patients: Beyond the "Actuarial versus clinical" assessment debate, Psychiatric Services, 59, 2, 184.

Burgelin J.-F., 2005, Santé, justice et dangerosités : pour une meilleure prévention de la récidive, Rapport de la Commission santé-justice, Paris, Ministère de la justice.

Bushway S. D., Morrison Pielh A., 2007, The inextricable link between age and criminal history in sentencing, Crime and delinquency, 53, 1, 156-183.

Campbell T. W., 2003, Sex offenders and actuarial risk assessments: ethical considerations. Behavioral Sciences \& Law, 21, 2, 269-279. 
Campbell T. W., DeClue G., 2010, Flying blind with naked factors: Problems and pitfalls in adjustedactuarial sex-offender risk assessment, Open Access Journal of Forensic Psychology, 2, 75-101.

Castel R., 1983, De la dangerosité au risque, Actes de la recherche en sciences sociales, 47, 119-127.

Chantraine G., Cauchie J.-F., 2006, Risque(s) et gouvernementalité, Socio-logos, Varia 1, 1-24.

Chiricos T., Welch K., Gertz, M., 2004, Racial typification of crime and support for punitive measures, Criminology, 42, 2, 359-390.

Commission Nationale Informatique et Libertés, 2011, Délibération n²001-232 du 21 juillet 2011 portant avis sur le projet de décret en Conseil d'État d'un traitement automatisé de données à caractère personnel dénommé "application des peines, probation et insertion" (APPI), publiée au Journal Officiel de la République Française $\mathrm{n}^{\circ} 259$ du 8 novembre 2011, texte ${ }^{\circ} 76$.

Conférence de Consensus sur la Récidive, 2013, Pour une nouvelle politique publique de prévention de la récidive, Principes d'actions et méthodes, Rapport du jury de consensus remis au Premier ministre, Paris.

Conseil de l'Europe, 2006, Recommandation Rec(2006)2 du Comité des ministres aux États membres sur les règles pénitentiaires européennes, adoptée le 11 janvier.

Conseil de l'Europe, 2010, Recommandation CM/REC(2010)1 du Comité des ministres aux États membres sur les règles du Conseil de l'Europe relative à la probation, adoptée le 20 janvier.

Conseil de l'Europe, 2014, Recommandation CM/REC(2014)3 du Comité des ministres aux États membres relative aux délinquants dangereux, adoptée le 19 février.

Cortoni F., Lafortune D., 2009, Le traitement correctionnel fondé sur des données probantes : une recension, Criminologie, 42, 1, 61-89.

Côté G., 2001, Les instruments d'évaluation du risque de comportements violents : mise en perspective, Criminologie, 34, 1, 31-45.

Crampagne S., 2013, L'évaluation de la dangerosité dans le cadre de l'expertise psychiatrique pénale, Thèse de doctorat en médecine, Faculté de médecine de Grenoble.

Crow M. S., 2008, The complexities of prior record, race, ethnicity, and policy, Interactive effects in sentencing, Criminal Justice Review, 33, 4, 502-523.

Crutchfield R. D., Skinner M. L., Haggerty K. P., McGlynn A., Catalano R. F., 2012, Racial disparity in police contacts, Race and Justice, 2, 3, 179-202.

Danet J., 2008, La dangerosité, une notion criminologique, séculaire et mutante, Champ pénal/Penal Field, vol. V.

Danet J. (dir.), 2013, La réponse pénale. Dix ans de traitement des délits, Rennes, PUR.

Delacrausaz P., Gasser J., 2012, La place des instruments d'évaluation du risque de récidive dans la pratique de l'expertise psychiatrique pénale : l'exemple lausannois, L'information psychiatrique, 2012, $88,439-443$.

Deprins D., 2006, La statistique, instrument de pouvoir ?, in Digneffe F., Moreau T. (dir.), La responsabilité et la responsabilisation dans la justice pénale, Bruxelles, Larcier/De Boeck, 501-522.

Dindo S., 2011, Sursis avec mise à l'épreuve : la peine méconnue, une analyse des pratiques de probation en France, Étude réalisée pour la DAP / Bureau PMJ1, Paris, ministère de la Justice.

Douglas K. S., Cox D. N., Webster C. D., 1999, Violence risk assessment: Science and Practice, Legal and criminological psychology, 4, 2, 149-184.

Douglas K. S., Skeem J. L., 2005, Violence Risk Assessment: Getting Specific About Being Dynamic, Psychology, Public Policy, and Law, 11, 3, 347-383.

Dozois J., Lalonde M., Poupart J., 1981, La dangerosité, un dilemme sans issue ?, Déviance et Société, 5, 383-401.

Dubourg É., (s.d.) Les Services Pénitentiaires d'Insertion et de probation, fondements juridiques, évolution, évaluation et avenir, Nantes, Thèse de droit et de sciences criminelles (en cours).

Du Mesnil du Buisson G., 2001, Quelles sont les implications juridiques de l'obligation de soin, injonction de soin, soin volontaire dans la prise en charge des auteurs d'agression sexuelle, in Fédération française de psychiatrie, Psychopathologie et traitements actuels des auteurs d'agression sexuelle, Conférence de consensus, Paris, John Libbey, Eurotext, 349-358.

Elbogen E. B., Mercado C. C., Scalora M. J., Tomkins, A. J., 2002, Perceived relevance of factors for violence risk assessment: a survey of clinicians, International Journal of Forensic Mental Health, 1, $37-47$. 
Ewald F., 1996, L'histoire de l'État-providence : les origines de la solidarité, Paris, Grasset et Festelle.

Farrington D. P., Jolliffe D., Johnstone L., 2008, Assessing Violence Risk: A Framework for Practice, Institute of Criminology, Cambridge University.

Feeley M., Simon J., 1992, The New Penology: Notes on the Emerging Strategy of Corrections and its applications, Criminology, 30, 449-474.

Ferri E., 1914, La sociologie criminelle, $2^{\mathrm{e}}$ éd., Paris, Alcan.

Forbes D., 2012, Oasys, dernière génération, Dedans/dehors, OIP, 76, 39-41.

Foucault M., 1981, L'évolution de la notion d' « individu dangereux » dans la psychiatrie légale, Déviance et Société, 5, 4, 403-22.

Freedman D., 2001, False prediction of future dangerousness: error rates and psychopathy checklistrevised, The Journal of the American Academy of Psychiatry and the Law, 29, 89-95.

Froment J.-C., Kaluszynski M. (dir.), L'administration pénitentiaire face aux principes de la nouvelle gestion publique, Grenoble, Cerdhap, PUG.

Garraud J.-P., 2006, Réponses à la dangerosité, Rapport sur la mission parlementaire confiée par le Premier ministre sur la dangerosité et la prise en charge des individus dangereux.

Gautron V., 2014, L'impact des préoccupations managériales sur l'administration locale de la justice pénale française, Champ pénal/Penal Field, Varia, XI.

Gautron V., Rétière J.-N., 2013, Des destinées judiciaires pénalement et socialement marquées, in Danet J. (dir.), La réponse pénale. Dix ans de traitement des délits, Rennes, PUR, 211-251.

Gendreau P., Little T., Goggin C., 1996, A meta-analysis of adult offender recidivism : What works!, Criminology, 34, 575-607.

Giovannangeli D., Cornet J-P., Mormont C., 2000, Étude comparative dans les 15 pays de l'Union Européenne : les méthodes et les techniques d'évaluation de la dangerosité et du risque de récidive des personnes présumées ou avérées délinquants sexuels, Université de Liège.

Gravier B., Lustenberg Y., 2005, L'évaluation du risque de comportements violents : le point sur la question, Annales Médico-Psychologiques, 163, 668-680.

Grove W. M., Meelh P. E., 1996, Comparative efficiency of informal (subjective, impressionistic) and formal (mechanical, algorithmic) prediction procedures: the clinical-statistical controversy, Psychology, Public, Policy and Law, 2, 293-323.

Guay J.-P., 2006, Prédiction actuarielle et prédiction clinique : le dernier souffle d'une pratique professionnelle, Revue internationale de criminologie et de police technique et scientifique, 2 , 49-164.

Guay J.-P., 2013, Évaluer le risque de récidive : un état de la recherche et des principaux enjeux pratiques, Conférence de consensus sur la prévention de la récidive, Contribution des experts, Paris.

Hannah-Moffat K., Maurutto P., 2003, Évaluation du risque et des besoins chez les jeunes contrevenants : un aperçu présenté à la division de la recherche et de la statistique, ministère de la Justice, Département de sociologie, Université de Toronto (Mississauga), Canada.

Hannah-Moffat K., 2013a, Punishment and risk, in Simon J., Sparks R. (eds), The Sage Handbook of Punishment and Society, London, Sage, 129-149.

Hannah-Moffat K., 2013b, Actuarial sentencing: an « unsettled » proposition, Justice Quarterly, 30, 2 , 270-296.

Hanson K. R., 2010, Les mêmes facteurs de risque prédisent la majorité des types de récidive, Recherche en Bref, Sécurité Publique du Canada, 15, 4.

Hanson K. R., Harris A. J. R., Scott T. L., Helmus L., 2007, Assessing the risk of sexual offenders on community supervision: The Dynamic Supervision Project, User Report, Ottawa, Public Safety Canada.

Hanson K. R., Morton-Bourgon G., Helmus L., Hodgson S., 2009, Méta-analyse de l'efficacité du traitement des délinquants sexuels : risque, besoin et réceptivité, Sécurité publique, Canada.

Hanson K. R., Thornton D., 1999, Statique-99 : une amélioration des évaluations actuarielles du risque chez les délinquants sexuels, Recherches correctionnelles, ministère du Solliciteur général du Canada.

Harcourt B. E., 2010, Risk as a proxy for race, University of Chicago, Law \& Economics, John M. Olin Working paper, 535 \& Public Law and Legal Theory Working paper, 323.

Harcourt B. E., 2011a, Surveiller et punir à l'âge actuariel. Généalogie et critique, part. I, Déviance et société, 35, 1, 5-33. 
Harcourt B. E., 2011b, Surveiller et punir à l'âge actuariel. Généalogie et critique, part. II, Déviance et société, 35, 2, 163-194.

Hare R. D., 1991, The Hare Psychopathy Checklist: Revised, Toronto, Ontario, Multi-Health Systems, Inc.

Hart S. D., Boer D. P., 2009, Structured professional judgment guidelines for sexual violence risk assessment: the Sexual Violence Risk-20 (SVR-20) and Risk for Sexual Violence Protocol (RSVP), in Otto R.K., Douglas K.S. (eds), Handbook of violence risk assessment, Oxford, Routledge, 269-294.

Hart S. D., Michie C., Cooke D. J., 2007, Precision of actuarial risk assessment instruments Evaluating the 'margins of error' of group v. individual predictions of violence, British Journal of psychiatry, suppl., $49,60-65$.

Hart S. D., Michie C., Cooke D. J., 2013, Another look at the (im-)precision of group v. individual predictions of violence, Behavioral Sciences and the Law, 31, 81-102.

Haute Autorité de Santé, 2011, Dangerosité psychiatrique : étude et évaluation des facteurs de risque de violence hétéro-agressive chez les personnes ayant des troubles schizophréniques ou des troubles de l'humeur, Audition Publique, Recommandations de la Commission d'audition, Paris.

Hayek F., 1953, Scientisme et sciences sociales : essai sur le mauvais usage de la raison, Paris, Plon.

Herzog-Evans M., 2012a, Outils d'évaluation : sortir des fantasmes et de l'aveuglément idéologique, Actualité Juridique Pénal, 2, 75-79.

Herzog-Evans M., 2012b, Exécution des peines, délinquance sexuelle et « positionnement quant aux faits » : enjeux juridiques et criminologiques, Actualité Juridique Pénal, 12, 632-635.

Hudson B., Bramhall G., 2005, Assessing the other: constructions of Asianness in risk assessment by probation officers, British Journal of Criminology, 45, 5, 721-740.

Inspection Générale des Finances, Inspection Générale des Services Judiciaires, 2011, Les services pénitentiaires d'insertion et de probation, Rapport, Paris.

Inspection des Services Pénitentiaires, 2011, Rapport relatif à la prise en charge de M. Tony Meilhon par le SPIP de Loire-Atlantique, Paris, Direction de l'Administration Pénitentiaire, ministère de la Justice.

Inspection des Services Pénitentiaires, 2013, Rapport relatif à l'utilisation du DAVC et aux pratiques d'évaluation des personnes placées sous main de justice, Paris, Direction de l'Administration Pénitentiaire, ministère de la Justice.

Jackson R. L., Hess D. T., 2007, Evaluation for civil commitment of sex offenders: A survey of experts, Sexual Abuse: A Journal of Research and Treatment, 19, 409-448.

Jendly M., 2012, Performance, transparence et accountability : une équation (dé) responsabilisante des professionnels exerçant en prison ?, Déviance et Société, 36, 3, 243-262.

Jobard F., Névanen S., 2007, La couleur du jugement. Discriminations dans les décisions judiciaires en matière d'infractions à agents de la force publique (1965-2005), Revue française de sociologie, 48, 2 , 243-272.

Jobard F., Lévy R., Goris I., 2009, Police et minorités visibles : les contrôles d'identité à Paris, New York, Open society Institute, Justice Initiative.

Kaluszynski M., 2008, Le retour de l'homme dangereux. Réflexions sur la notion de dangerosité et ses usages, Champ pénal/Penal Field, vol. V.

Kaluszynski M., 2013, La science pénitentiaire comme science de gouvernement. Espaces juridiques, réseaux réformateurs et savoirs experts en France à la fin du XIX ${ }^{\mathrm{e}}$ siècle, Revue d'anthropologie des connaissances, 7, 1, 87-111.

Kaminski D., 2009, Pénalité, Management, innovation, Namur, Presses universitaires de Namur.

Kensey A., Benaouda A., 2011, Les risques de récidive des sortants de prisons. Une nouvelle évaluation, Cahiers d'études pénitentiaires et criminologiques, Paris, Direction de l'Administration Pénitentiaire, $36,8 \mathrm{p}$.

Krauss D. A., Scurich N., 2013, Risk assessment in the Law: Legal admissibility, scientific validity and some disparities between research and practice, Behavioral Sciences and the Law, 31, 215-229.

Kropp P. R., Hart S., Webster C., Eaves D., 1999, Spousal Risk Assessment guide user's manual, Toronto, ON, Canada, Multi-Health Systems and BC Institute Against Family Violence.

Lalande P., 2006, Punir ou réhabiliter les contrevenants ?, Du « Nothing Works » au «What Works » (Montée, déclin et retour de l'idéal de réhabilitation), in Lalande P., Lamalice O. (dir.), La sévérité pénale à l'heure du populisme, ministère de la Sécurité Publique, Canada, 30-77 
Lamanda V., 2008, Amoindrir les risques de récidive des condamnés dangereux, Rapport à M. le président de la République.

Lameyre X., 2001, Pour une éthique des soins pénalement obligés, Revue de science criminelle et de droit pénal comparé, 3, 521-536.

Larminat (de) X., 2012, La probation en quête d'approbation. L'exécution des peines en milieu ouvert entre gestion des risques et gestion des flux, Thèse de science politique, CESDIP, Guyancourt, Université de Versailles Saint Quentin.

Larminat (de) X., 2013a, La technologie de mise à distance des condamnés en France. La centralisation informatique des données socio-judiciaires, Déviance et Société, 37, 3, 359-373.

Larminat (de) X., 2013b, La probation en quête d'approbation : du consensus politique à l'aveuglement positiviste, Archives de Politique Criminelle, 35, 45-60.

Larminat (de) X., 2014a, Hors des murs. L'exécution des peines en milieu ouvert, Paris, PUF.

Larminat (de) X., 2014b, Un continuum pénal hybride. Discipline, contrôle, responsabilisation, Champ Pénal/Penal Field, XI.

Lazerges C., 2012, Le choix de la fuite en avant au nom de la dangerosité, Les lois 1, 2, 3, 4, 5, etc. sur la prévention et la répression de la récidive, Revue de science criminelle et de droit pénal comparé, $1,274-279$.

Lhuillier D. (dir.), 2007, Changements et constructions des identités professionnelles : les travailleurs sociaux pénitentiaires, PsyForm, 125.

Lund C. A., 2000, Predictors of sexual recidivism: did meta-analysis clarify the role and relevance of denial?, Sexual Abuse : a journal of research and treatment, 12, 4, 275-287.

Lussier P., Davies G., 2011, A person-oriented perspective on sexual offenders, offending trajectories, and risk of recidivism: A new challenge for policymakers, risk assessors, and actuarial prediction?, Psychology, Public Policy, and Law, 17, 4, 530-561.

Manzanera C., Senon J.-L., 2008, L'expertise psychiatrique pénale, in Senon J.-L., Lopez G., Cario R. (dir.), Psycho-criminologie, Paris, Dunod, 147-162.

Martel J., Brassard R., Jaccoud M., 2011, When two worlds collide risk management in Canadian Aboriginal corrections, British Journal of Criminology,51, 2, 235-255.

Martinson R., 1974, Whats Works?, Questions and answers about prison reform, The Public Interest, $35,22-54$.

Mary P., 2001, Pénalité et gestion risque ; vers une justice « actuarielle » en Europe, Déviance et société, $25,1,33-51$.

McGrath R. J., Cumming G. F., Burhcard S. Z., Ellerby L., 2010, Current practices and emerging trends in sexual abuser management: The Safer Society 2009 North American Survey, Brandon (Vt.), Safer Society Press.

McSherry B., 2013, Managing Fear: The Law and Ethics of Preventive Detention and Risk Assessment, Oxford, Routledge.

Milburn P., Jamet, L., 2013, La prévention de la récidive comme secteur de l'action institutionnelle : processus d'ajustements entre acteurs, normes et pratiques, Recherche réalisée avec le soutien de la Mission de recherche Droit et Justice, Paris, GIP, Rapport final de recherche.

Millaud F., Dubreucq J.-L., 2012, Les outils d'évaluation du risque de violence : avantages et limites, L'information Psychiatrique, 88, 6, 431-437.

Mills J. F., Kroner D. G., 2006, The effect of discordance among violence and general recidivism risk estimates on predictive accuracy, Criminal Behaviour and Mental Health, 16, 155-166.

Ministère de la Justice, 2011, Rapport du groupe de travail sur le fonctionnement des Services Pénitentiaires d'Insertion et de Probation, Direction de l'Administration Pénitentiaire, Ministère de la Justice.

Monahan J., 1981, Predicting violent behavior: An assessment of the clinical techniques, Beverly Hills (CA), Sage.

Monahan J., 1997, Clinical and Actuarial Predictions of Violence (from modern scientific evidence : the law and science of expert testimony), in Faigman D., Kaye D., Saks M., Sanders J. (eds), From modern scientific evidence: the law and the science expert testimony, St. Paul, Minnesota, West Publishing Corporation, 1, 300-318. 
Morash M., 2009, A great debate over using the Level of Service Inventory-revised (LSI-R) with women offenders, Criminology and Public Policy, 8, 173-181.

Mossman D., 2013, Evaluating risk assessments using receiver operating characteristic analysis: Rationale, advantages, insights, and limitations, Behavioral Sciences \& the Law, 31, 1, 23-39.

Moulin V., Palaric R., Gravier B., 2012, Quelle position professionnelle adopter face à la diversité des problèmes posés par l'évaluation des dangerosités ?, L'information Psychiatrique, 2012, 88, 8, 617-629.

Muller C., Wildeman C., 2013, Punishment and Inequality, in Simon J., Sparks R. (eds), The Sage handbook of Punishment and Society, London, Sage, 169-185.

Newbold K., 2011, An evaluation of the offender assessment system as an assessment tool for the national probation service, Internet Journal of criminology, 1-36.

Niveau G., 2011, Évaluation de la dangerosité et du risque de récidive, Paris, L’Harmattan.

Nunes K. L., Hanson R. K., Firestone P., Moulden H. M., Greenberg D. M., Bradford J. M., 2007, Denial predicts recidivism for some sexual offenders, Sexual Abuse: a journal of research and treatment, 9, 2, 91-105.

Observatoire National de la Délinquance et des Réponses Pénales, 2012, La criminalité en France, Rapport, Paris, éd. CNRS.

Oleson J. C., 2011, Risk in sentencing: Constitutionally-Suspect Variables and Evidence-Based Sentencing, Southern Methodist University Law Review, 64, 1329-1404.

Ottenhof R., Favard A.M. (dir.), 2001, L'exécution par l'Administration Pénitentiaire des mesures en milieu ouvert, Rapport final, Université de Nantes, GIP, Mission de recherche Droit et Justice.

Padfield N., 2010, La condamnation, la gestion et le traitement des délinquants « dangereux », rapport final, Strasbourg, Comité Européen pour les problèmes criminels, Conseil de coopération pénologique.

Pager D., 2003, The Mark of a Criminal Record, American Journal of sociology, 108, 5, 937-975.

Pager D., 2008, The Republican Ideal? Ethnic Minorities and the Criminal Justice System in Contemporary France, Punishment and Society, 10, 4, 375-400.

Parent G., Guay J.-P., Bernard C., Knight R. A., 2009, Intégration sociale et récidive chez les délinquants sexuels graves : une étude exploratoire, in Tardif M. (dir.), L'agression sexuelle : transformations et paradoxes, Cifas, 256-283.

Péchillon E., 2011, Administration Pénitentiaire et performance budgétaire, in Froment J.C., Kaluszynski M. (dir.), L'administration pénitentiaire face aux principes de la nouvelle gestion publique, Grenoble, Cerdhap, PUG, 91-102.

Proulx J., Lussier P., 2001, La prédiction de la récidive chez les agresseurs sexuels, Criminologie, 34, $1,9-29$.

Quinsey V. L., Harris G. T., Rice G. T., Cormier, C. A., 1998, Violent offenders: Appraising and managing risk, Washington, DC, American Psychological Association.

Quirion B., D'Addese L., 2011, De l'évaluation clinique au calcul de probabilité : le recours aux outils actuariels dans les pénitenciers canadiens, Criminologie, 44, 2, 225-250.

Quirion B., Jendly M., Vacheret M., 2012, Le système pénal et la (dé)responsabilisation des acteurs, Déviance et société, 36, 3, 235-241.

Raynor P., Lewis S., 2011, Risk-need assessment, sentencing and minority ethnic offenders in Britain, British Journal of Social Work, 41, 7, 1357-1371.

Reichman N., 1986, Managing crime risks: Toward an insurance-based model of social control, Research in Law, Deviance and Social Control, 8, 151-172.

Robinson R., 2002, Exploring risk management in probation practice, contemporary developments in England and Wales, Punishment \& society, 4, 1, 5-25.

Rutter M., 1985, Resilience in the face of adversity: protective factors and resistant to psychiatric disorder, British Journal of Psychiatry, 185, 147, 598-611.

Saleilles R., 1898, L’individualisation de la peine, Étude de criminalité sociale, Paris, Alcan.

Sarre E., 2001, Beyond «What Works? », A 25 year jubilee retrospective of Robert Martinson famous article, Australian and New Zealand journal of criminology, 34, 1, 38-46.

Senon J.-L., 2012, La psychiatrie à l'épreuve de l'insécurité sociale : la dangerosité ou plutôt la prédiction du risque de violence en toile de fond du débat psychiatrie-justice, L'Information psychiatrique, 88, 6, 407-414. 
Senon J.-L., Pascal J.-C., Rossinelli G., (dir.), 2007, Expertise psychiatrique pénale, Audition publique des 25 et 26 janvier 2007, Fédération Française de Psychiatrie, Montrouge, John Libbey Eurotext.

Senon J.-L., Voyer M., Paillard C., Jaafari N., 2009, Dangerosité criminologique : données contextuelles, enjeux cliniques et expertaux, L'information psychiatrique, 85, 8, 719-725.

Silver E., Miller L. L., 2002, A Cautionary Note on the Use of Actuarial Risk Assessment Tools for Social Control, Crime \& Delinquency, 8, 1, 138-161.

Simmons J. P., Nelson L. D., Simonsohn U., 2011, False-positive Psychology: Undisclosed Flexibility in Data Collection and Analysis Allows Presenting Anything as Significant, Psychological Science, XX, $\mathrm{X}, 1-8$.

Singh J. P., Fazel S., 2010, Forensic risk assessment: a metareview, Criminal Justice and Behavior, 37, 965-988.

Singh J. P., Grann M., Fazel S., 2011, A comparative study of risk assessment tools: a systematic review and metaregression analysis of 68 studies involving 25,980 participants, Clinical Psychology Review, $31,3,499-513$

Singh J. P., Grann M., Fazel S., 2013, Authorship Bias in Violence Risk Assessment? A Systematic Review and Meta-Analysis, Plos one, 8(9): e72484. doi:10.1371/journal.pone.0072484.

Singh J. P., Fazel S., Gueorguieva R., Buchanan A., 2014, Rates of violence in patients classified as high risk by structured risk assessment instruments, British Journal of Psychiatry, 3, 180-187.

Slingeneyer T., 2007, La nouvelle pénologie, une grille d'analyse des transformations des discours, des techniques et des objectifs dans la pénalité, Champ pénal/Penal Field, IV, Varia.

Starr S. B., 2014, Evidence-Based Sentencing and the Scientic Rationalization of Discrimination, Stanford law review, 66, 803-872.

Szmukler G., 2001, Violence risk prediction in practice, The British Journal of Psychiatry: the Journal of Mental Science, 178, 84-85.

Szmukler G. M. D., Rose N., 2013, Risk Assessment in Mental Health Care: Values and Costs, Behavioral Sciences \& the Law, 31, 1, 125-140.

Tabary A., 2005, L'enquête sociale dans le cadre judiciaire. De la saisine par le juge au rapport écrit du travailleur social, Paris, L'Harmattan.

Vacheret M., 2010, Sciences criminologiques, peines de prison et professionnels, Revue de science criminelle et de droit pénal comparé, 4, 983-987.

Vacheret M., Cousineau M. M., 2005, L'évaluation du risque de récidive au sein du système correctionnel canadien : regards sur les limites d'un système, Déviance et société, 29, 4, 379-397.

Vacheret M., Dozois J., Lemire G., 1998, Le système correctionnel canadien et la nouvelle pénologie la notion de risque, Déviance et socief́é, 22, 1, 1998, 37-50.

Van Voorhis P., Wright E., Salisbury E., Bauman A., 2010, Women's risk factors and their contributions to existing risk/needs assessment: The current status of a gender-responsive supplement, Criminal Justice and Behaviour, 37, 3, 261-288.

Vogel (de) V., Vries Robbé (de) M., De Ruiter C., Bouman Y., 2011, Assessing protective factors in forensic psychiatric practice: introducing the SAPROF, International Journal of Forensic mental health, 10, 171-177.

Vogelvang B., Tigges L., 2012, Qu'est ce qui « marche » et « ne marche pas » pour prévenir la récidive dans le cadre de la probation ?, in Mbanzoulou P., Herzog-Evans M., Courtine S. (dir.), Insertion et désistance des personnes placées sous main de justice. Savoirs et pratiques, Paris, L'Harmattan, 199-241.

Voyer M., Senon J.-L., 2012, Présentation comparative des outils d'évaluation du risque de violence, L'information psychiatrique, 88, 6, 445-453.

Vries Robbé (de) M., Vogel (de) V., De Spa E., 2011, Protective factors for violence risk in forensic psychiatric patients: a retrospective validation study of the SAPROF, International Journal of Forensic mental health, 10, 178-186.

Vries Robbé (de) M., Vogel (de) V., Stam J., 2012, Protective factors for violence risk: the value for clinical practice, Psychology, 3, $\mathrm{n}^{\circ} 12 \mathrm{~A}, 1259-1263$.

Vrieze S. I., Grove W. M., 2010, Multidimensional assessment of criminal recidivism: problems, pitfalls, and proposed solutions, Psychological assessment, 22, 2, 382-395.

Wakefield S., Uggen C., 2010, Incarceration and Stratification, Annual Review of Sociology, 36, 387-406. 
Webster C. D., Douglas K. S., Eaves D., Hart S. D., 1997, HCR-20, Évaluation du risque de violence, Version 2, Burnaby BC, Simon Fraser University.

\section{Notes}

1 Depuis la loi n²004-204 du 9 mars 2004 portant adaptation de la justice aux évolutions de la criminalité, de nombreuses lois ont traité de la question de la récidive, parmi lesquelles figurent notamment la loi $\mathrm{n}^{\circ}$ 2005-1549 du 12 décembre 2005 relative au traitement de la récidive des infractions pénales, la loi $\mathrm{n}^{\circ}$ 2007-1198 du 10 août 2007 renforçant la lutte contre la récidive introduisant les peines planchers ; la loi $\mathrm{n}^{\circ} 2008-174$ du 25 février 2008 relative à la rétention de sûreté et à la déclaration d'irresponsabilité pénale; la loi n ${ }^{\circ}$ 2010-242 du 10 mars 2010 tendant à amoindrir le risque de récidive criminelle.

$2 \mathrm{Au}$ titre des dispositions réglementaires figure principalement la circulaire DAP n ${ }^{\circ} 113 / \mathrm{PMJ} 1 \mathrm{du} 19$ mars 2008 relative aux missions et aux méthodes d'intervention des SPIP et la circulaire du 8 novembre 2011 relative au diagnostic à visée criminologique (DAVC).

3 Exigées par le législateur dans un nombre croissant de situations, préalablement à l'obtention d'une mesure d'aménagement de peine, au prononcé ou à la levée d'une mesure de sûreté (surveillance judiciaire, surveillance de sûreté, rétention de sûreté), le nombre d'expertises psychiatriques a augmenté de plus de $149 \%$ entre 2002 et 2009 (Blanc, 2011, 83).

4 Circulaire DAP n $113 / \mathrm{PMJ} 1$ du 19 mars 2008 relative aux missions et aux méthodes d'intervention des SPIP.

5 Décret n²005-445 du 6 mai 2005 modifiant le décret $n^{\circ}$ 93-1114 du 21 septembre 1993 relatif au statut particulier du personnel d'insertion et de probation de l'Administration Pénitentiaire et le décret n ${ }^{\circ} 99-670$ du 2 août 1999 relatif au statut d'emploi du directeur des services pénitentiaires, NOR : JUSK0540009D, art. 5.

6 L'un des CPIP mobilise notamment le questionnaire élaboré par l'Institut National de Prévention et d'Éducation pour la Santé pour évaluer le niveau de consommation d'alcool et établir le niveau de dépendance. Voir [http://www.inpes.sante.fr/cfesbases/catalogue/pdf/861.pdf].

7 Circulaire JUSK1340021C du 6 août 2013 relative à la sécurité dans les SPIP.

8 Note DAP 000104 du 13 juillet 2010 relative aux enquêtes de faisabilité dans le cadre du placement sous surveillance électronique.

9 Préalablement condamné à de multiples reprises principalement pour des faits de vols mais également de violences et d'agression sexuelle, M. Tony Meilhon a été condamné le 30 juin 2009 par le tribunal correctionnel de Nantes à une peine d'un an d'emprisonnement dont six mois assortis d'un sursis avec mise à l'épreuve pendant deux ans pour des faits d'outrage à magistrats en récidive. Pris en charge par l'antenne du milieu fermé du SPIP de Loire-Atlantique pendant sa période de détention, il a été libéré en fin de peine le 24 février 2010. Il devait alors être pris en charge par l'antenne du milieu ouvert de ce service chargé de mettre en œuvre le SME, cette mesure prenant fin le 24 février 2012. Mais son dossier n'a pas été effectivement affecté, entraînant une absence de suivi en pratique. En janvier 2011, Tony Meilhon commet un meurtre particulièrement sordide sur une jeune fille pendant sa période de sursis.

10 Conseil d'État, Décision n`355624 du 11 avril 2014.

11 Les cadres des SPIP A et $\mathrm{C}$ faisaient notamment partie de ce groupe de travail.

12 Circulaire du 8 novembre 2011 relative au diagnostic à visée criminologique, NOR : JUS1140051C.

13 Circulaire du 8 novembre 2011, 2.5 .

14 Voir sur ce point : CNIL, 2011, Délibération $n^{\circ} 2001-232$ du 21 juillet 2011 portant avis sur le projet de décret en Conseil d'État d'un traitement automatisé de données à caractère personnel dénommé "application des peines, probation et insertion" (APPI), publiée au Journal Officiel de la République Française $\mathrm{n}^{\circ} 259$ du 8 novembre 2011 , texte $\mathrm{n}^{\circ} 76$.

15 Rapport définissant les objectifs de la politique d'exécution des peines, Annexe à la loi n²012-409 du 27 mars 2012 de programmation relative à l'exécution des peines, publiée au Journal Officiel de la République Française du 28 mars 2012, p. 5592, texte, 1, II.A.3.

16 Étude d'impact, Annexe au projet de loi $\mathrm{n}^{\circ} 1413$ relatif à la prévention de la récidive et à l'individualisation des peines enregistré à la Présidence de l'Assemblée nationale le 9 octobre 2013, 108.

\section{Pour citer cet article}

Référence électronique 
Émilie Dubourg et Virginie Gautron, «La rationalisation des méthodes d'évaluation des risques de récidive », Champ pénal/Penal field [En ligne], Vol. XI I 2014, mis en ligne le 18 novembre 2014, consulté le 18 décembre 2015. URL : http://champpenal.revues.org/8947 ; DOI : 10.4000/ champpenal.8947

\section{$\grave{A}$ propos des auteurs}

\section{Émilie Dubourg}

Doctorante en droit pénal et sciences criminelles à l'université de Nantes, rattachée au laboratoire Droit et changement social (UMR 6028). Contact : emiliedubourg@ hotmail.com

Virginie Gautron

Maître de conférences (droit) à l'université de Nantes, chercheuse au laboratoire Droit et changement social (UMR6297). Contact : virginie.gautron@univ-nantes.fr

\section{Droits d'auteur}

CC Champ pénal

\section{Résumés}

L'évaluation des justiciables revêt une importance essentielle dans le processus judiciaire de détermination de la peine. Elle permet d'individualiser la sanction au regard de leur profil et de leur situation. L'individualisation de la peine se joue désormais non plus tant au stade de son prononcé qu'au stade de son exécution, conférant un rôle important aux Conseillers Pénitentiaires d'Insertion et de Probation chargés du suivi des condamnés. Leurs méthodes d'évaluation, originellement cliniques et faiblement structurées, ont été remises en cause, au regard d'instruments plus structurés, voire actuariels, principalement élaborés et mis en œuvre dans les pays anglo-saxons. Si la recherche d'une meilleure structuration du jugement professionnel des agents de probation apparaît pertinente, il convient toutefois d'interroger les différentes méthodes d'évaluation, leur fiabilité prédictive et leur impact en termes de prise en charge des justiciables. Le recours à des instruments plus structurés, a fortiori actuariels, opère en effet un glissement des finalités de l'évaluation, en l'inscrivant non plus tant dans une logique de réinsertion que dans une logique de gestion des risques de récidive.

The assessment of offenders is crucial in the judicial process of sentencing. It allows the individualization of the sanction according to their profile and situation. Today, this individualization is mostly realized through the execution of the sentence, rather than at the stage of sentencing. This confers an important role to the probation officers responsible for the management of sentenced individuals. Originally clinical and weakly structured, their assessment methods have now been questioned by the rise of more structured, even actuarial, instruments, mainly developed in Anglo-Saxon countries. If the search for a stronger structuring of probation officers' professional judgment seems relevant, it is nevertheless imperative to question the various contemporary assessment approaches, their predictive reliability and their impact on the management of condemned individuals. The reliance on more structured instruments, especially actuarial instruments, produces a shift in the ends of assessment, which is less concerned with reintegration than with managing the risk of reoffending.

\section{Entrées d'index}

Mots-clés : évaluation, jugement professionnel, instruments actuariels, risque, récidive Keywords : assessment, professional judgment, actuarial instruments, risk, reoffending

Géographique : France

Chronologique : XXle siècle, années 2000, années 2010 